\title{
What constrains distributional learning in adults?
}

\author{
Dave F. Kleinschmidt ${ }^{*} \dagger$
}

June 4, 2020

\begin{abstract}
One of the many remarkable features of human language is it's flexibility: during acquisition, any normally-developing human infant can acquire any human language, and during adulthood, language users quickly and flexibly adapt to a wide range of talker variation. Both language acquisition in infants and adaptation in adults have been hypothesized to be forms of distributional learning, where flexibility is driven by sensitivity to statistical properties of sensory stimuli and the corresponding underlying linguistic strucutres. Despite the similarities between these forms of linguistic flexibility, there are obvious differences as well, chief among them being that adults have a much harder time acquiring the same unfamiliar languages that they would have picked up naturally during infancy. This suggests that there are strong constraints on distributonal learnign during adulthood. This paper provides further, direct evidence for these constraints, by showing that American English listeners struggle to learn voice-onset time (VOT) distributions that are atypical of American English. Morever, computational modeling shows that the pattern of distributional learning (or lack thereof) across different VOT distributions is consistent with Bayesian belief-updating, starting from prior beliefs that are very similar to the VOT distributions produced by a typical talker of American English. Together, this suggests that distributional learning in adults is constrained by prior experience with other talkers, and that distributional learning may be a computational principle of human language that operates throughout the lifespan.
\end{abstract}

\section{Introduction}

A basic fact of human language is that any typically developing human infant can learn any human language. Human languages vary dramatically at every level, including the basic sound systems they use, and the human language faculty must be flexible enough to deal with this substantial cross-linguistic variability. The first stages of language acquisition are characterized by initial flexibility, which declines over development as the particulars of the native language are acquired. For instance, as infants become better at discriminating linguistically important sounds in their native language, they simultaneously lose the ability to discriminate some sounds that are important for other languages but not their native language (Catherine T. Best, McRoberts, LaFleur, \& Silver-Isenstadt, 1995; Kuhl, Williams, Lacerda, Stevens, \& Lindblom, 1992; Werker \& Tees, 1984; but see Catherine T Best, McRoberts, \& Sithole, 1988). Ultimately, people become sufficiently inflexible over development that they generally struggle to learn another language in adulthood (Hartshorne, Tenenbaum, \& Pinker, 2018).

However, adult listeners still need to deal with substantial variability within their native language, as talkers differ in how they realize the phonetic categories of the language using acoustic cues (e.g. Allen, Miller, \& DeSteno, 2003; Clopper, Pisoni, \& de Jong, 2005; Newman, Clouse, \& Burnham, 2001). Accordingly, adult listeners flexibly adapt to unfamiliar talkers in a wide variety of contexts. At one extreme, perception of heavily accented non-native talkers becomes faster and more accurate with just a few minutes of exposure (Baese-berk, Bradlow, \& Wright, 2013; Bradlow \& Bent, 2008; Clarke-Davidson \& Garrett, 2004). At the other extreme, listeners recalibrate representations of individual phonetic categories based on subtle changes in single segments in otherwise unaccented talkers (Bertelson, Vroomen, \& de Gelder, 2003; Kraljic \& Samuel, 2006; Norris, McQueen, \& Cutler, 2003).

\footnotetext{
${ }^{*}$ Corresponding author: dave.kleinschmidt@rutgers.edu; 152 Frelinghuysen Ave, Piscataway NJ, 08854

${ }^{\dagger}$ Rutgers Unversity (New Brunswick, NJ, USA) and University of Rochester (Rochester, NY, USA)
} 
Both acquisition and adaptation have been theorized to be forms of distributional learning. First, at a computational level (in the sense of Marr, 1982), acquisition can be treated straightforwardly as a problem of distributional learning. The probabilistic relationships between different linguistic and sensory units define the structure of the language, and so learning the structure of your language requires learning - at least approximately and implicitly - the distributions associated with different structures in the language. Likewise, accommodating talker variability is also, at a computational level, a problem of learning the speaker- or context-specific probabilistic relationships between observable cues and underlying linguistic structures. This similarity is demonstrated by the computational modeling which shows that both acquisition (Feldman, Griffiths, Goldwater, \& Morgan, 2013; McMurray, Aslin, \& Toscano, 2009; Vallabha, McClelland, Pons, Werker, \& Amano, 2007; but see Hitczenko, Mazuka, Elsner, \& Feldman, 2018) and rapid adaptation (Kleinschmidt \& Jaeger, 2015; Theodore \& Monto, 2019) can be treated as forms of distributional learning.

Second, both adults and infants are sensitive to distributional properties of speech. One set of findings shows that listeners (both infants and adults) become more sensitive after exposure to a bimodal distribution of an acoustic cue (like length, voice-onset time, vowel formant frequencies, etc.) compared with exposure to a unimodal distribution (e.g. Escudero, Benders, \& Wanrooij, 2011; Feldman, Myers, White, Griffiths, \& Morgan, 2013; Goudbeek, Cutler, \& Smits, 2008; Maye \& Gerken, 2000; Maye, Werker, \& Gerken, 2002), although the evidence that very young infants can benefit from this kind of exposure in short laboratory sessions is equivocal (Cristià, 2018). Another set of findings shows that adult listeners can adapt to changes in the means and/or variances of the cue distributions for known phonetic categories (e.g. Chládková, Podlipský, \& Chionidou, 2017; Clayards, Tanenhaus, Aslin, \& a Jacobs, 2008; Colby, Clayards, \& Baum, 2018; Schreiber, Onishi, \& Clayards, 2013; Theodore \& Monto, 2019; Theodore, Myers, \& a. Lomibao, 2015). What both of these sets of findings have in common is that listeners pick up on the distributions of cues without any explicit instruction about the intended category label associated with each token. For example, Clayards et al. (2008) had listeners listen to /b/-/p/ minimal pair words (e.g., "beach/peach") with different voice-onset times (VOT), and click on a matching picture to indicate which member of the minimal pair they heard. On every trial, the VOT was drawn from one of two bimodal distributions, which had clusters with the same means but different variances across subjects. Listeners in the high-variance condition produced shallower categorization functions, reflecting greater uncertainty associated with the wider range of VOTs they heard for each cluster.

If both acquisition and adaptation can be treated as forms of distributional learning, and both infants and adults are sensitive to distributional information, what distinguishes acquisition from adaptation? For one, it seems that distributional learning in adults is constrained. Adult listeners struggle to learn new categories that are not present in their native language. For instance, Japanese listeners struggle to discriminate the English /r/-/l/ contrast, which corresponds a single category in their native language (Goto, 1971; Miyawaki et al., 1975). Long-term naturalistic exposure is not sufficient to achieve good discrimination of this contrast, even after conversational competence has been achieved (Takagi \& Mann, 1995). While perception of this contrast can be improved somewhat by training, it requires extensive training and these listeners seldom achieve native-like levels of performance (Bradlow, Pisoni, Akahane-Yamada, \& Tohkura, 1997).

There are also apparent constraints on the ability of adult listeners to adapt to variations in the distributions associated with native language categories. For instance, Idemaru and Holt (2011) tested how well listeners adapt to distributions of two cues that distinguish voicing (e.g., /b/ vs. /p/), voice onset time (VOT, the primary cue to voicing) and the pitch of the following vowel (f0, a secondary cue). These two cues are typically positively correlated in English, with /p/ corresponding to high VOT and high f0, and /b/ to low values of both cues (Kohler, 1982). In one condition, listeners were exposed to a talker who produced a positively correlated distribution of these cues. During a post-test, these listeners used f0 to categorize stops with ambiguous VOTs. In another condition, listeners heard a talker who produced an uncorrelated distribution, where f0 is uninformative. In contrast to the listeners in the first condition, during the post test these listeners ignored f0 even for ambiguous VOTs. This effect is consistent with the idea that listeners are rationally integrating multiple cues to voicing, weighing them based on how informative they are (Bejjanki, Clayards, Knill, \& Aslin, 2011; Clayards et al., 2008; Ernst \& Bülthoff, 2004). However, listeners in a third condition who were exposed to a talker who produced an anticorrelated distribution did not follow the predictions of rational cue integration. Despite the fact that $\mathrm{f} 0$ was just as informative for this accent as for the positively correlated accent, listeners ignored f0 as a cue to voicing. This suggests that these listeners have ruled out the possibility of a reversed mapping between $\mathrm{f} 0$ and voicing (/b/ vs. /p/), possibly perhaps 
American English talkers typically do not typically produce it (e.g., House, 1953). Likewise, Sumner (2011) found that American English listeners had trouble adapting to a talker who produced VOT distributions for /b/ and /p/ that had substantially lower means (approximately $-60 \mathrm{~ms}$ and $0 \mathrm{~ms}$, respectively) than a typical American English talker (approximately 0-10ms and 60ms VOT; Lisker \& Abramson, 1964).

So on the one hand, distributional learning provides a unifying theoretical perspective on flexibility in language acquisition and adaptation. On the other hand, it highlights an important difference between these two basic kinds of plasticity in the language system. From the lens of distributional learning, one major difference between acquisition and adaptation is that distributional learning in adulthood appears to be constrained, while it is relatively unconstrained during acquisition.

However, we lack a clear understanding of the nature and source of those constraints. There are a number of other differences between the learning problems posed by acquisition and adaptation, even if both are forms of distributional learning. First, distributional learning in infancy is, at least initially, almost entirely unsupervised, meaning that there is very little direct information about whether any two observed sounds come from the same cluster (category) or different ones. Any such information to this effect that $i s$ available comes from context that these sounds occur in, like the fact that sounds are embedded in words, which infants begin to learn early, in parallel with the phonetic categories of their language (Bergelson \& Swingley, 2012). While this kind of "self-supervision" can serve as an important additional source of information in distributional learning (Feldman, Griffiths, et al., 2013; Feldman, Myers, et al., 2013), it is much weaker than the richer, less uncertain circumstantial evidence that adults can draw on from the lexicon, pragmatic context, phonotactics, etc. These contextual sources often provide a great deal of information about the intended category for a particular cue value, which makes the distributional learning problem of adaptation often (but not always) effectively a supervised learning problem.

Second, when adapting to an unfamiliar talker, adults have a great deal of prior experience with other talkers which they could use to narrow down the possible distributions they ought to expect (Kleinschmidt \& Jaeger, 2015). Both of these factors might contribute to constraints on adult distributional learning. For the first, if adult adaptation typically operates in a supervised setting, the fully unsupervised setting of a typical distributional learning experiment might not provide enough information, leading to reduced learning. For the second, if the distributions encountered in an experiment fall far enough outside the range of what a listener expects based on their prior experience, they may struggle to adapt (Kleinschmidt \& Jaeger, 2015).

The goal of this paper is to systematically probe the constraints on distributional learning in American English-speaking adults. Experiment 1 tests the ability of American English listeners to change their classification of word initial stop voicing based on experience with a range of distributions of voice-onset time (VOT). The results of this experiment show that distributional learning by adults is, in fact, constrained, and moreover these constraints are qualitatively consistent with prior experience with typical talkers of American English. Experiment 2 tests an alternative possible constraint on distributional learning, which is the absence of labels, which could lead to uncertainty about whether the bimodal distribution really corresponds to the standard English categories of voiced and voiceless stops of $/ \mathrm{b} /$ and $/ \mathrm{p} /$. Surprisingly, telling listeners whether a particular VOT was intended to be a voiced /b/ or a voiceless $/ \mathrm{p} /$ on half of the trials has no effect on the speed or completeness of distributional learning. Experiment 3 uses a Bayesian belief-updating model to test whether the constraints observed in Experiment 1 can be explained as belief updating starting from a common set of prior beliefs that is shared by all of the subjects. Finally, Experiment 4 tests distributional learning for a wider range of VOT distributions, and the results suggest that the constraints on distributional learning may be more complex than revealed by Experiments 1-3.

Together, these results show that distributional learning in adults is constrained, and these constraints are at least consistent with belief updating starting from a set of prior beliefs about the VOT distributions that a typical talker of American English will produce.

\section{Experiment 1: Distributional learning of voicing from VOT}

In order to probe the possible constraints on distributional learning, Experiment $1^{1}$ measures listener's distributional learning of a range of bimodal distributions of voice-onset time (VOT). These distributions

\footnotetext{
${ }^{1}$ The data from the experiments reported in this paper is available downloaded via the supunsup $\mathrm{R}$ package, and the $\mathrm{R}$ code to reproduce these analyses (in the form of an RMarkdown file) can be found at osf.io/tv8wm/.
} 


\section{Experiment 1: VOT distribution conditions}

Similarity of exposure talker to typical talker controlled by shifting means of $/ \mathrm{b} /$ and $/ \mathrm{p} /$ distributions

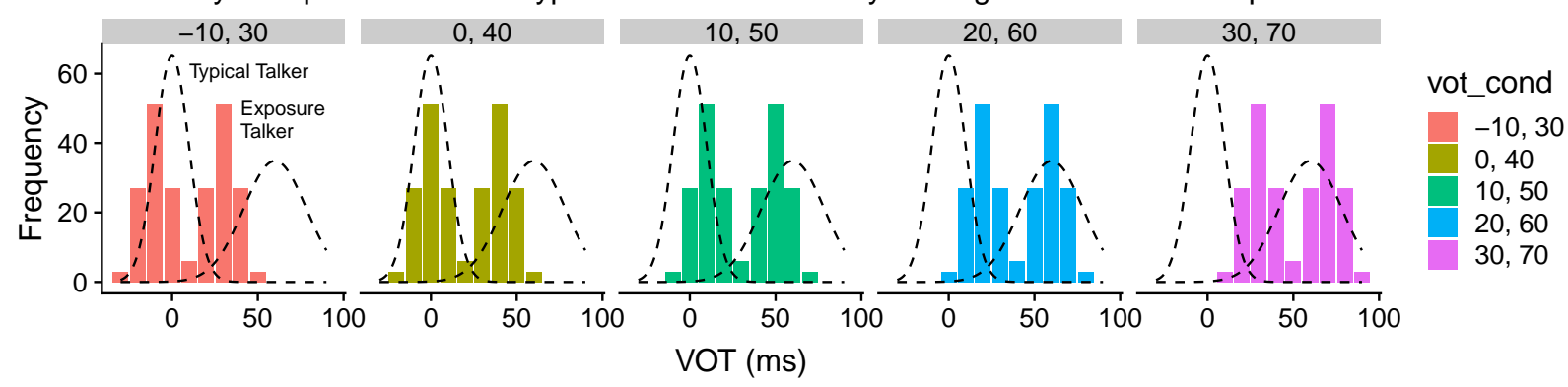

Figure 1: Each subject heard one of these five synthetic accents, which differ only in the distribution of VOTs of the word-initial stops. Black dashed lines show VOT distributions from a hypothetical typical talker (as estimated by Kronrod, Coppess, \& Feldman, 2016). Note that the 0 and 10ms shifted accents are reasonably close to this typical talker, while the $-10,20$, and 30ms shifted accents deviate substantially.

vary in how similar they are to a typical American English talker (Figure 1). Each distribution has the same variance and separation between the VOT clusters $(40 \mathrm{~ms})$, but the overall VOTs are shifted up or down in $10 \mathrm{~ms}$ increments. Previous results have shown that listeners are sensitive to such shifts in distributions (Munson, 2011), but have not systematically explored the wide range of shifts used here. While some of these VOT distributions deviate substantially from American English, they are not outside the range of variation across other languages (see e.g. Cho, Whalen, \& Docherty, 2019; Lisker \& Abramson, 1964). Thus, if distributional learning by these adult listeners is unconstrained, their category boundaries should be close to the optimal category boundaries for the VOT distributions they were exposed to.

\subsection{Methods}

\subsubsection{Participants}

155 participants were recruited via Amazon's Mechanical Turk. Participants were paid $\$ 2.00$ for participation, which took about 20 minutes. Participants were excluded who participated more than once $(n=1)$ or who failed to classify VOTs reliably ( $n=17 ; n=1$ for both reasons). Reliable classification is defined as accuracy of at least $80 \% / \mathrm{b} /$ at 0 and $80 \% / \mathrm{p} /$ at $70 \mathrm{~ms}$ VOT. Because some conditions had few stimuli with these VOTs, participants' responses were extrapolated using a logistic generalized linear model (GLM). Excluded participants were roughly equally distributed across conditions (maximum of 5 in $10 \mathrm{~ms} / \mathrm{b} /$ VOT condition, and minimum of 1 in $20 \mathrm{~ms} / \mathrm{b} / \mathrm{VOT}$ condition). After these exclusions, data from 138 participants remained for analysis.

\subsubsection{Procedure}

The procedure is based on Clayards et al. (2008). Figure 2 shows an example trial display. On each trial, two response option images appeared, which corresponded to one of three /b/-/p/ minimal pairs (beach/peach, bees/peas, or beak/peak). Participants started each trial by clicking on a button between the two pictures, which played the corresponding minimal pair word audio stimulus. Participants then clicked on the picture to indicate whether they heard the $/ \mathrm{b} /$ or $/ \mathrm{p} /$ member of the minimal pair. Participants performed 222 of these trials, evenly divided between the three minimal pairs, in random order.

Each trial's word was synthesized with a voice onset time (VOT) that was randomly drawn from a bimodal distribution, with low and high VOT clusters implicitly corresponding to /b/ and /p/, respectively. This distribution defined the accent that the subject heard, and each subject was pseudorandomly assigned to one of five accent conditions (Fig. 1). 

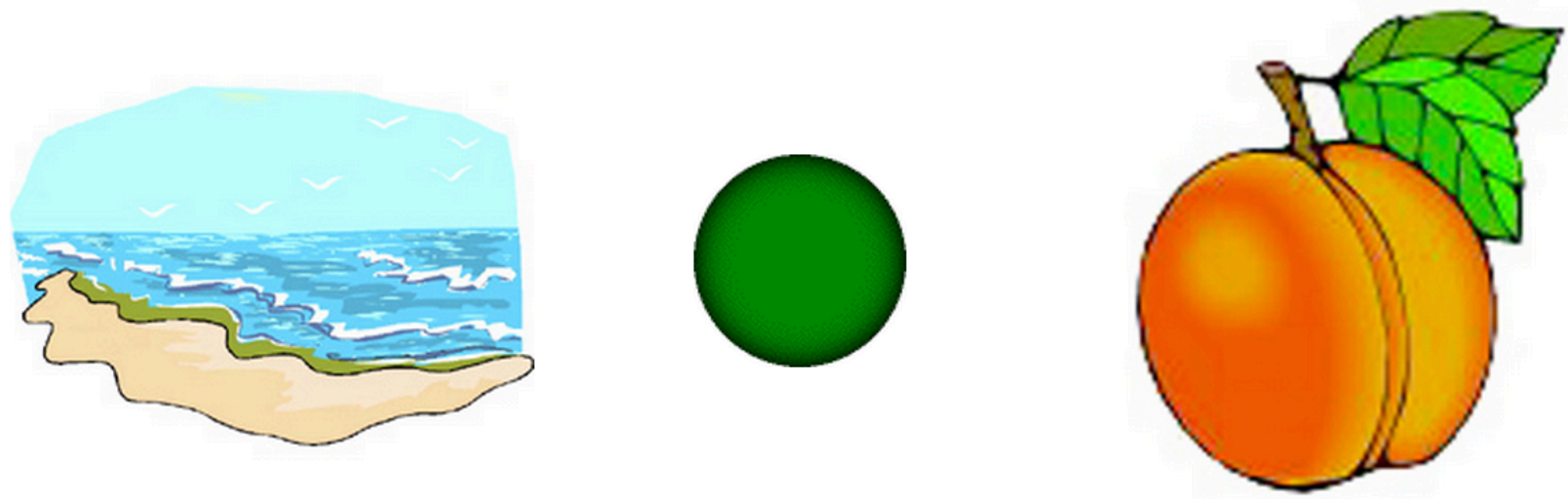

Figure 2: Example trial display (beach/peach). Listeners first click on the green button to play the word, then click on one picture to indicate what they heard.

\subsubsection{Materials}

The audio and visual stimuli were identical to those in Clayards et al. (2008). Three /b/-/p/ minimal pair audio continua were synthesized using the 1988 Klatt synthesizer (Klatt, 1980), by manipulating VOT in $10 \mathrm{~ms}$ increments (either adding voicing before the stop burst to create negative VOTs, or aspiration after for positive VOTs). Within a /b/-/p/ continuum, the other parameters were held constant, and modeled on natural tokens of the endpoints (beach/peach, bees/peas, and beak/peak).

\subsection{Results}

In order to assess distributional learning, each subject's classification function is compared to two baselines, corresponding to no learning and complete learning. The no learning baseline is derived from the category boundary for a typical talker's VOT distributions (based on Kronrod, Coppess, \& Feldman, 2016), and the category boundary based solely on the exposure talker's distributions. I refer to the typical talker's boundary as the "no learning" baseline, and the exposure talker's boundary as the "complete learning" baseline.

Each subject's classification function was estimated with a Bayesian multilevel (mixed-effect) logistic regression, using the brms package (Bürkner, 2017) for $\mathrm{R}$ ( $\mathrm{R}$ Core Team, 2020). ${ }^{2}$ This approach simultaneously estimates the group-level effects of the experimental manipulation (VOT shift condition) on the /b/-/p/ category boundary location and sharpness, and each individual participants' boundary locations and slopes. A multilevel approach like this has the benefit of properly accounting for and balancing the joint uncertainty about the group- and individual-level effects (Gelman \& Hill, 2007). ${ }^{3}$ A specific advantage of a Bayesian approach, over standard mixed-effects models as fit with 1me4, is that the Bayesian approach allows for direct estimates of subject-level effects (i.e., category boundaries), with associated uncertainty.

The first question is whether listeners learned anything at all from exposure to these VOT distributions. Figure 3 shows that, qualitatively, listeners generated different categorization functions after exposure to

\footnotetext{
${ }^{2}$ The model formula was respP $\sim 1+$ bvotCond $*$ vot_s * trial_s + (1 + vot_s I subject), where respP is a binary variable with 0 for a /b/ response and 1 for /p/, bvotCond is the exposure condition (treatment-coded), and vot_s and trial_s are the centered and scaled VOT and number of each trial, respectively.

${ }^{3}$ One effect of using a multilevel approach is that the estimate of each subject's boundary is "shrunk" towards the group-level estimate. This reflects the assumption that participants are drawn from the same population, and hence the data from one subject in a group are informative about other participants in that group (and vice versa). The amount of this shrinkage depends on the amount of data available from each individual subject, and the consistency of the individual subject estimates. In this case, each subject contributes sufficient data to make shrinkage mild, and an alternative analysis that estimates each participants' boundary with a separate logistic GLM produces qualitatively similar effects.
} 


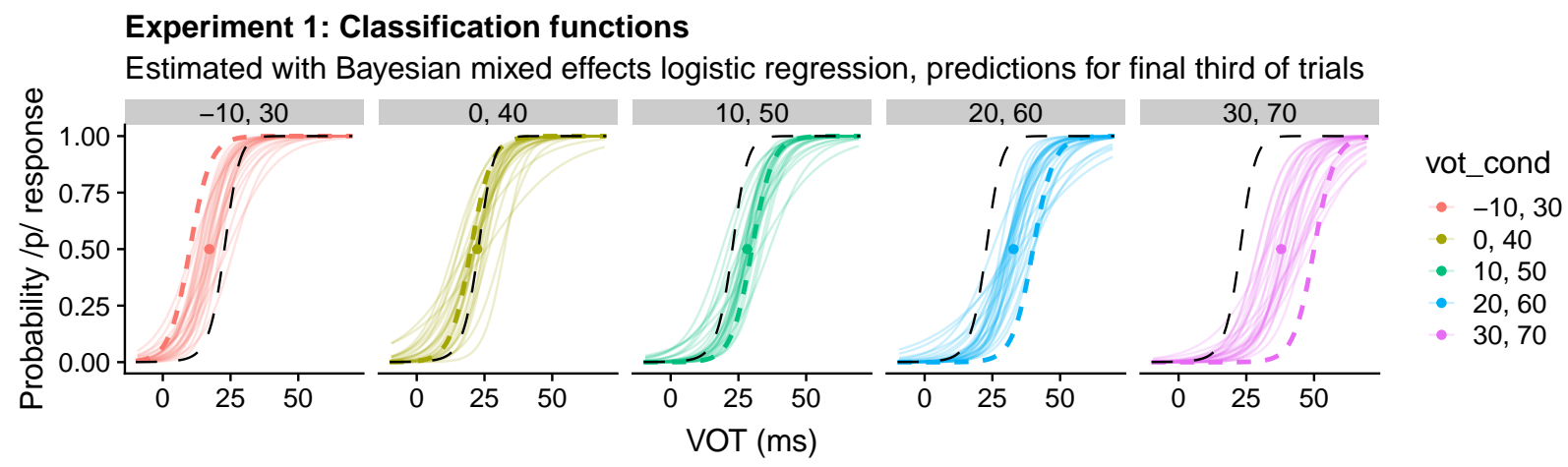

Figure 3: Results from experiment 1 show that distributional learning is constrained, with individual subjects' classification functions (fine colored lines) representing an apparent compromise between the ideal boundary given a typical talker's distributions (skinny, black dashed lines), and the ideal boundary for the exposure distributions (heavy colored dashed lines; see fig. 1 for both). Subjects' classification functions were estimated for the final third of trials (i.e., at trial 185 out of 222) based on the posterior distributions of the fixed and random effects. Dots show the estimated population boundary in each condition, based on the fixed effects alone.

different distributions. The results of the Bayesian mixed-effects regression verify this qualitative impression, with the overall probability of responding /p/ increasing from each condition to the next with $p>0.986 .{ }^{4}$

A more informative way of answering this question is to look at the category boundary - the VOT that is ambiguous between $/ \mathrm{b} /$ and $/ \mathrm{p} /$ - corresponding to the fixed effects for each exposure condition. Because trial number was included as a regressor, trial was fixed at 184 ( $83 \%$ or $5 / 6$ of the 222 total trials). The large white points in Figure 4 show the estimated boundary for each condition, and the corresponding confidence intervals are the $95 \%$ posterior intervals (e.g., the $95 \%$ quantiles of the posterior samples). Visual inspection shows that the group-level posterior distributions of the boundaries are almost entirely non-overlapping with their most-similar/neighboring conditions (all $p<0.001$ ), even though the distributions of individual subjects' boundaries do overlap (as shown by the colored violins).

The second question is how completely listeners learned the distributions they were exposed to. Visual inspections of the categorization functions fig. 3 and the category boundaries fig. 4 suggests that listeners category boundaries (at $83 \%$ through the exposure) do not perfectly correspond to the ideal category boundary for the VOT distributions they were exposed to (thick dashed colored lines). Specifically, listeners seem to undershoot the ideal category boundary, and their categorizations functions lie between the ideal boundary for the exposure talker and the boundary corresponding to a typical talker of American English (thin black lines).

The undershoot is largest in absolute terms for the most shifted distributions, with the boundary for the $30,70 \mathrm{~ms}$ VOT condition undershooting by $12 \mathrm{~ms}(95 \% \mathrm{CI}[14,10])$ and the $-10,30 \mathrm{~ms}$ condition by $7 \mathrm{~ms}(95 \%$ CI $[5,9])$.

\subsection{Discussion}

These results indicate two things. First, like many other studies (e.g. Chládková et al., 2017; Clayards et al., 2008; Colby et al., 2018; Theodore \& Monto, 2019; Theodore et al., 2015), I found evidence for distributional learning by adults: listeners do change how they categorize sounds as voiced or unvoiced due to exposure to different VOT distributions. Second, this learning is not complete, in the sense that listeners' voicedunvoiced boundary does not exactly match the boundary implied by the bimodal distribution they were

\footnotetext{
${ }^{4}$ The $p$ values reported here are Bayesian $p$ values, or the posterior probability that the relevant statement holds conditional on the data and model. These are determined using samples from the posterior, by computing the proportion of those samples where the statement is true. In this case, the relevant condition is whether the category boundary for one condition is reliably less than the boundary for the condition with a next highest mean VOTs.
} 


\section{Experiment 1: Category boundaries}

Compared with no learning and complete learning

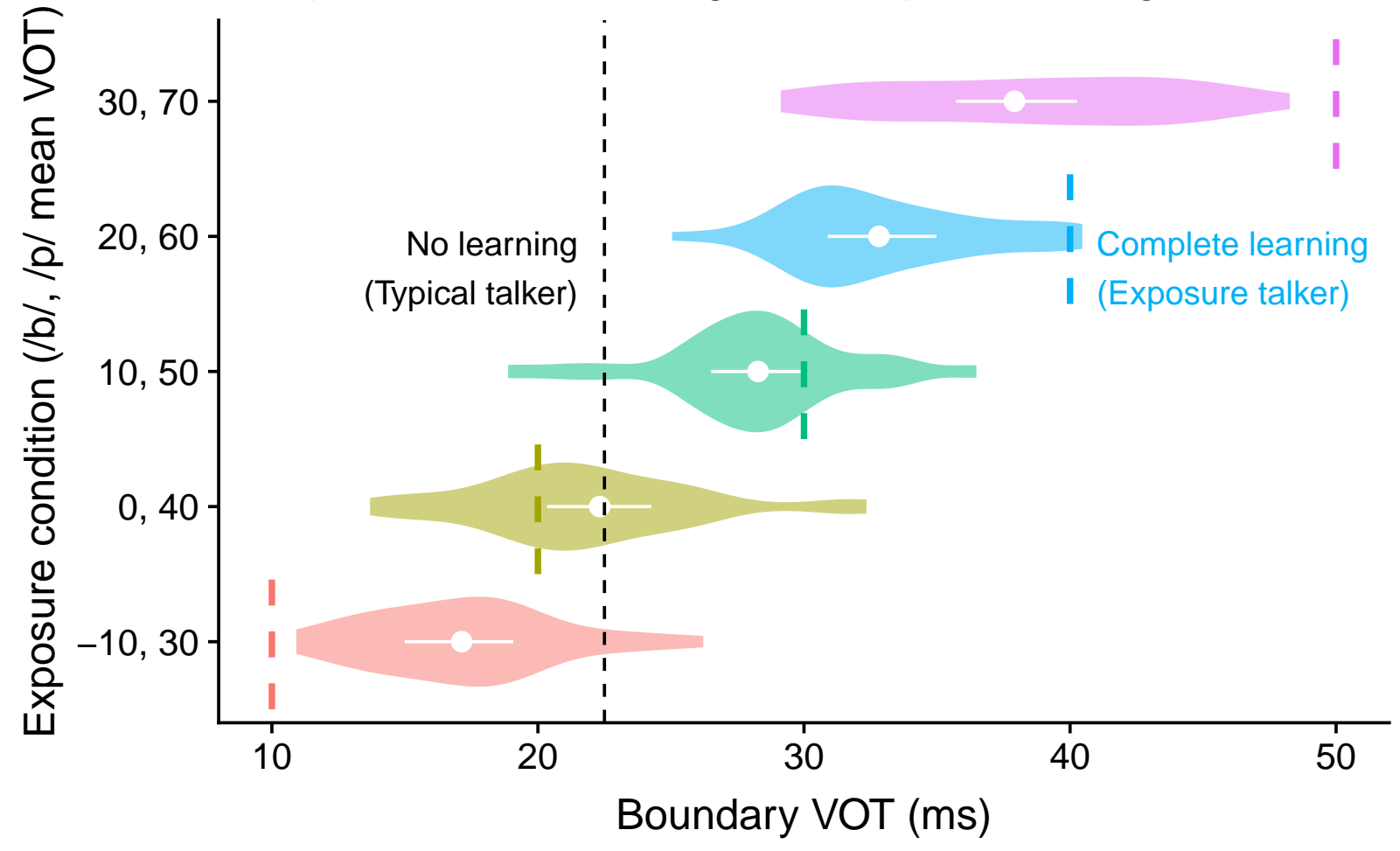

Figure 4: Category boundaries by condition. Violins show the distribution of participants' boundaries (random effects), and the white points show the group-level boundary (fixed effects, with 95\% Bayesian credible intervals). The black dashed line shows the ideal category boundary given a typical talker's distributions, and the colored heavy dashed lines show the ideal boundaries based on each condition's exposure distributions (see fig. 1). 
exposed to. Like the findings of Sumner (2011) and Idemaru and Holt (2011), this second result suggests that distributional learning is constrained somehow. In the following experiments, I investigate two possible sources of these constraints.

First, it is possible that the primary constraint here is that listeners are being asked to do unsupervised distributional learning. That is, they are free to interpret each VOT they hear as either a /b/ or a /p/, since both options are available for a response. For distributions that are highly shifted, most tokens will fall into one of the two pre-existing distributions (see Fig. 1): in the condition with the highest positive shift $(30 \mathrm{~ms} / \mathrm{b} /, 70 \mathrm{~ms} / \mathrm{p} /), 73 \%$ of all tokens from the lower cluster would be classified as $/ \mathrm{p} / \mathrm{according}$ to a typical talker's distributions. Thus, what was intended to be two separate clusters of VOTs - one for /b/ and one for /p/ - may instead have been interpreted by listeners as one large cluster in the extreme conditions, lacking any labels to tell them otherwise. I address this possibility in Experiment 2, by providing labels on a portion of trials.

A second possibility is that listeners are constrained by their prior experience with other talkers. The fact that listeners' category boundaries tend to lie between those of a typical talker and the experimental exposure talker suggests that this is not implausible. To assess this possibility more rigorously, in Experiment 3 I use a Bayesian belief-updating model of distributional learning to investigate whether the pattern of distributional learning across conditions is consistent with belief updating starting from common prior beliefs.

\section{Experiment 2: Semi-supervised distributional learning}

In this experiment, some trials are presented with label information, which indicates which member of the /b/-/p/ minimal pair the speaker intended to produce. The goal of this experiment is to investigate whether the constraints on distributional learning observed in Experiment 1 are due to the unsupervised nature of the distributional learning task. Adding labels to some of the trials might improve the degree of learning, the speed of learning, or both.

\subsection{Methods}

\subsubsection{Procedure}

The procedure was identical to that of Experiment 1, with one change. Half of the trials were unlabeled as in Experiment 1: pictures for both the $/ \mathrm{b} /$ and $/ \mathrm{p} /$ members of the minimal pair for that trial's word were available (e.g., "beach" and "peach"). The other half of the trials were labeled: if the word was drawn from a "beach" to "peach" continuum and the VOT was sampled from the lower VOT cluster, than one response option would be "beach", and the other either "peak" or "peas". That is, the only response that was compatible with the rest of the word had an onset consistent with the VOT cluster that that trial was drawn from.

Two different semi-supervised conditions were run which differed in how labeled and unlabeled trials were distributed across VOTs (Fig. 5). No differences were found between behavior in these conditions so they are analyzed together. ${ }^{5}$

The only other difference with Experiment 1 was that the $-10 \mathrm{~ms}, 30 \mathrm{~ms}$ condition was not included. This is because these two experiments were originally run concurrently as a pilot for an imaging study, during which the $-10 \mathrm{~ms}$, 30ms condition was not considered. The original goal of the supervised conditions of Experiment 2 was to see if distributional learning could be accelerated by including some labeled trials. The $-10 \mathrm{~ms}, 30 \mathrm{~ms}$ condition of Experiment 1 was run later, after analyzing data from the other conditions Experiments 1 and 2 together.

\subsubsection{Participants}

A total of 249 participants were recruited via Amazon's Mechanical Turk. As in Experiment 1, participants were paid $\$ 2.00$ for participation. Exclusion criteria were the same as Experiment 1, and data from 16 participants were excluded from analysis: 4 for repeated participation (including participants who had

\footnotetext{
${ }^{5}$ For the curious - or skeptical — reader, the two conditions are listed separately in the supunsup R data package that accompanies this paper.
} 


\section{Experiment 2: semi-supervised learning}

\section{Distribution of labeled trials in two semi-supervised learning conditions}

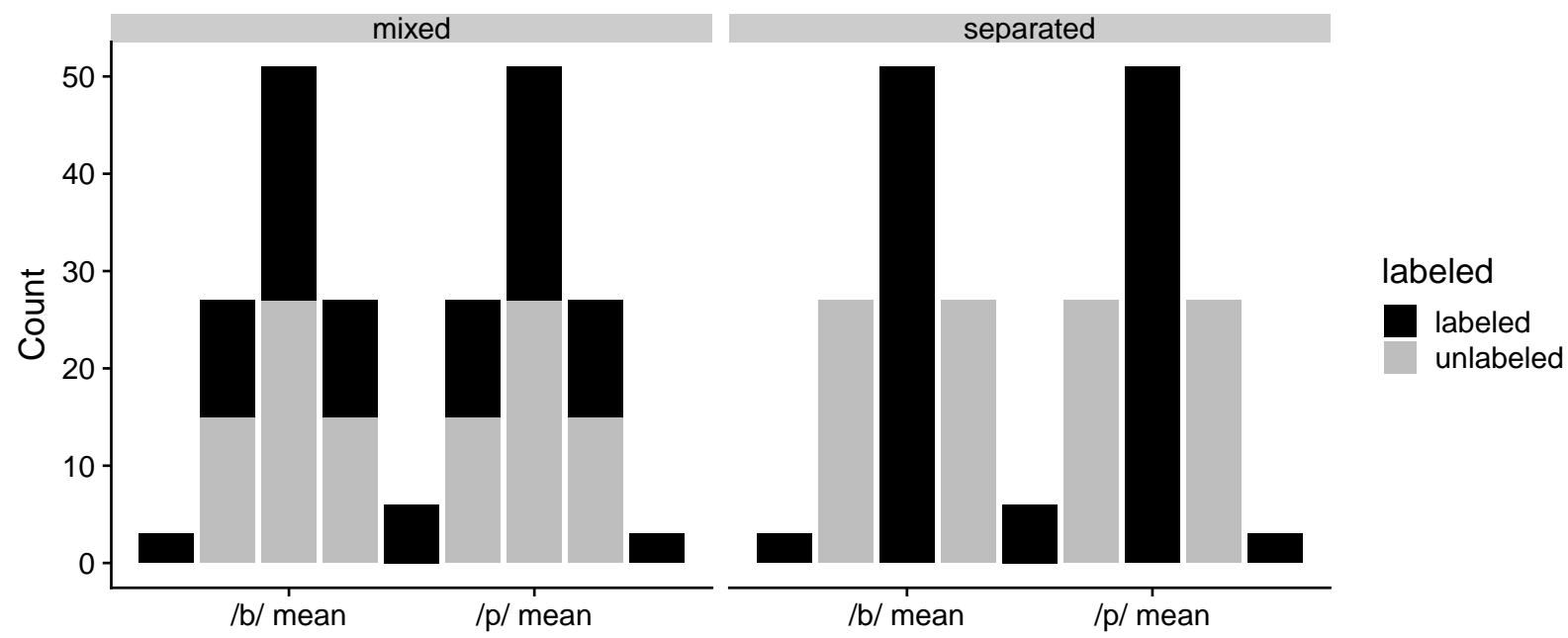

Figure 5: Distribution of labeled and unlabeled trials in Experiment 2. These conditions have the same number of labeled and unlabeled trials, but differ in how they are assigned to VOTs. In the "mixed" condition, most VOTs occurred in a mixture of labeled and un-labeled trials. In the "separated" condition, each VOT always occurred as either labeled or unlabeled. Because no difference was found between learning in these conditions, they are analyzed together.

participated in Experiment 1), 13 for inaccurate categorization of unambiguous VOTs, with 1 excluded for both criteria. This left data from 233 remaining for analysis. ${ }^{6}$ The larger sample size reflects the fact that there were originally two different variants of the supervised learning task, which yielded similar results and are thus analyzed together here.

\subsection{Results}

First, listeners were highly sensitive to the labels on labeled trials, responding consistent with the label $98 \%$ of the time (range across participants of $72-100 \%$ ).

Second, did listeners use labels to guide or accelerate their learning? As with Experiment 1 I assessed listeners' distributional learning via category boundaries estimated with a Bayesian logistic mixed-effects model. This model included data from both Experiments 1 and 2 in order to directly assess effects of supervised learning on the strength and time course of learning. This model included fixed effects for supervision condition, all fixed effects from the Experiment 1 model, and all interactions thereof. The random effects structure was identical to Experiment $1 .^{7}$ Because listeners' responses were so systematically determined by the label on labeled trials, only unlabeled trials were included in the model (all trials from Experiment 1, and half of the trials from Experiment 2).

Figure 7 shows the by-subject and overall category boundaries for semi-supervised (shaded violins/points) and unsupervised (outlined violins/points, re-plotted from Figure 4) distributional learning. As in Fig. 4, the boundaries estimated from the model for a trial $5 / 6$ of the way to the end of the experiment, and the

\footnotetext{
${ }^{6}$ The full datasets for all experiments reported here, including subjects excluded from analysis, is available in the supunsup $\mathrm{R}$ package.

${ }^{7}$ The model formula was respP $\sim 1+$ bvotCond $*$ supervised $*$ vot_s $*$ trial_s $+(1+$ vot_s $\mid$ subject $)$. As in Experiment 1 , VOT and trial number were centered and scaled to have zero mean and unit variance. Default treatment contrasts were used for supervised and bvotCond, with the base levels being supervised and /b/ mean VOT of 0ms, respectively. Default priors were used except for the addition of a Student's $t$ prior for the regression coefficients $(\beta \mathrm{s})$ with 3 degrees of freedom, mean 0 , and scale of 1 (student_t $(3,0,1)$ in the brms syntax); for discussion of this see the supplementary material.
} 


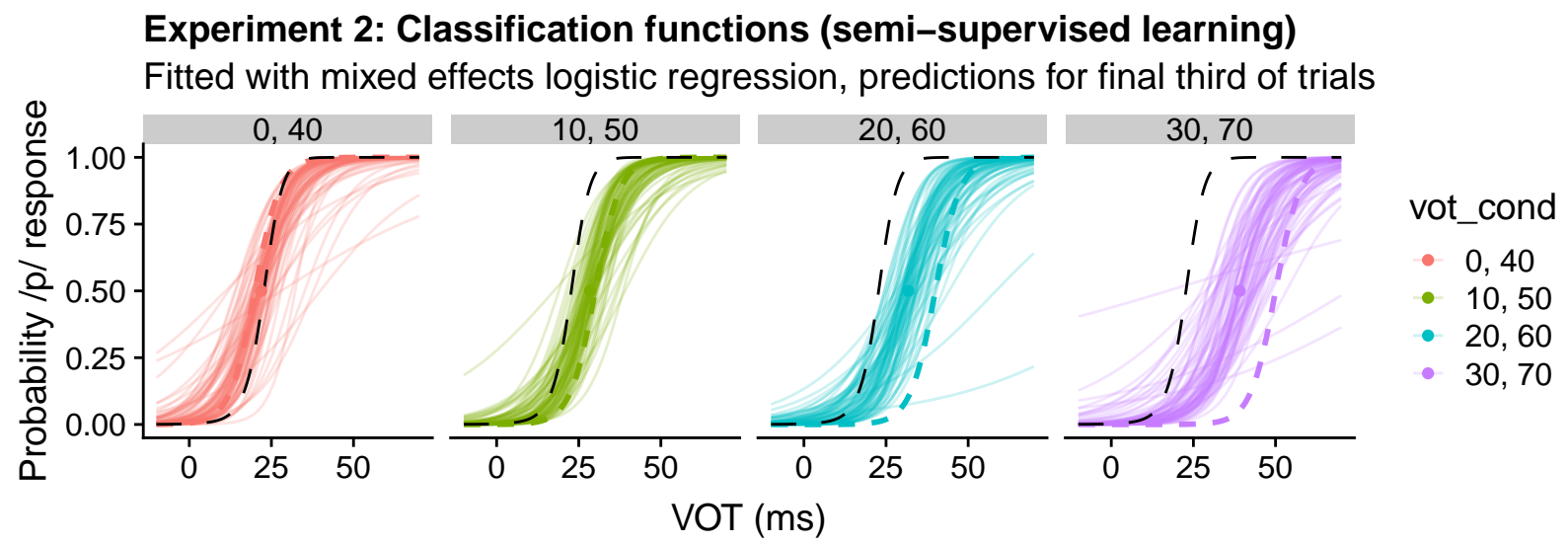

Figure 6: Classification functions from semi-supervised distributional learning. As for Experiment 1 (Figure 3), individual subjects categorization functions (fine colored lines) are intermediate between the ideal boundary given a typical talker's distributions (skinny, black dashed lines), and the ideal boundary for the exposure distributions (heavy colored dashed lines).

points and lines show the group-level (fixed effects) estimates and $95 \%$ credible intervals. The intervals for unsupervised and semi-supervised overlap for all VOT conditions, indicating that the inclusion of labeled trials makes no reliable difference in distributional learning, at least at the trial where these boundaries are estimated.

Similarly, by a variety of measures, the estimates of the regression coefficients themselves suggest that the addition of labeling information did not change listeners distributional learning. First, the $95 \%$ credible intervals for all fixed effects coefficients involving the supervision condition include 0. Second, the Bayes Factors (BFs) for the null hypotheses that each of the regression coefficients involving the supervision condition is exactly zero suggest that the data provide either weak $(1<B F<3.2,6$ out of 16 predictors) or "substantial" ( $B F \geq 3.2,10$ of 16) evidence in favor of the null (using the somewhat arbitrary cutoff of Kass \& Raftery, 1995). By both standards, the addition of labeling information has little to no effect on the overall amount of learning in each condition individually, does not affect category boundary steepness (interactions with VOT), and does not change the speed of learning speed (interactions with trial number).

Based on the category boundaries themselves, the condition that comes the closest to showing any effect is the $30 \mathrm{~ms} / \mathrm{b} /, 70 \mathrm{~ms} / \mathrm{p} / \mathrm{VOT}$, which is the most extreme shift relative to standard VOT distributions. However, the boundaries in this condition are highly variable across subjects, and moreover the grouplevel boundaries in this condition may have been biased by the presence of three outlier participants with unreliable responses which leads to very shallow classification functions and very high estimated boundaries (greater than 50ms; see Figure 6). These participants weren't so unreliable as to be excluded by the pre-set exclusion criteria so they are included in the analysis here for the sake of completeness. Even so, there is still no significant (Bayesian $p<0.05$ ) effect of supervision in any regression coefficient or the estimated boundary, and the single-subject boundary distribution for this condition (violins in Fig. 7) are very similar.

\subsection{Discussion}

The results of Experiment 2 suggest that labeling individual VOTs as /b/ or /p/ does very little to affect listeners' distributional learning. Both at the condition level (fixed effect boundaries) and the subject level (distribution of participants' boundaries), distributional learning leads to the same shift in category boundaries regardless of whether all trials are unlabled (Experiment 1) or half labeled (Experiment 2). This in turn suggests that the discrepancy between listeners' learned boundaries and the boundary implied by the exposure distribution is not due to uncertainty about the whether any particular VOT was intended to be a $/ \mathrm{b} /$ or a $/ \mathrm{p} /$. This is somewhat surprising, given that in the extremely shifted $30 \mathrm{~ms} / \mathrm{b} /, 70 \mathrm{~ms} / \mathrm{p} /$ condition most of the tokens from the $/ \mathrm{b} /$ distribution would normally be classified as $/ \mathrm{p} /$ under a typical talker's 


\section{Experiment 2: Semi-supervised adaptation Compared with unsupervised (Experiment 1)}

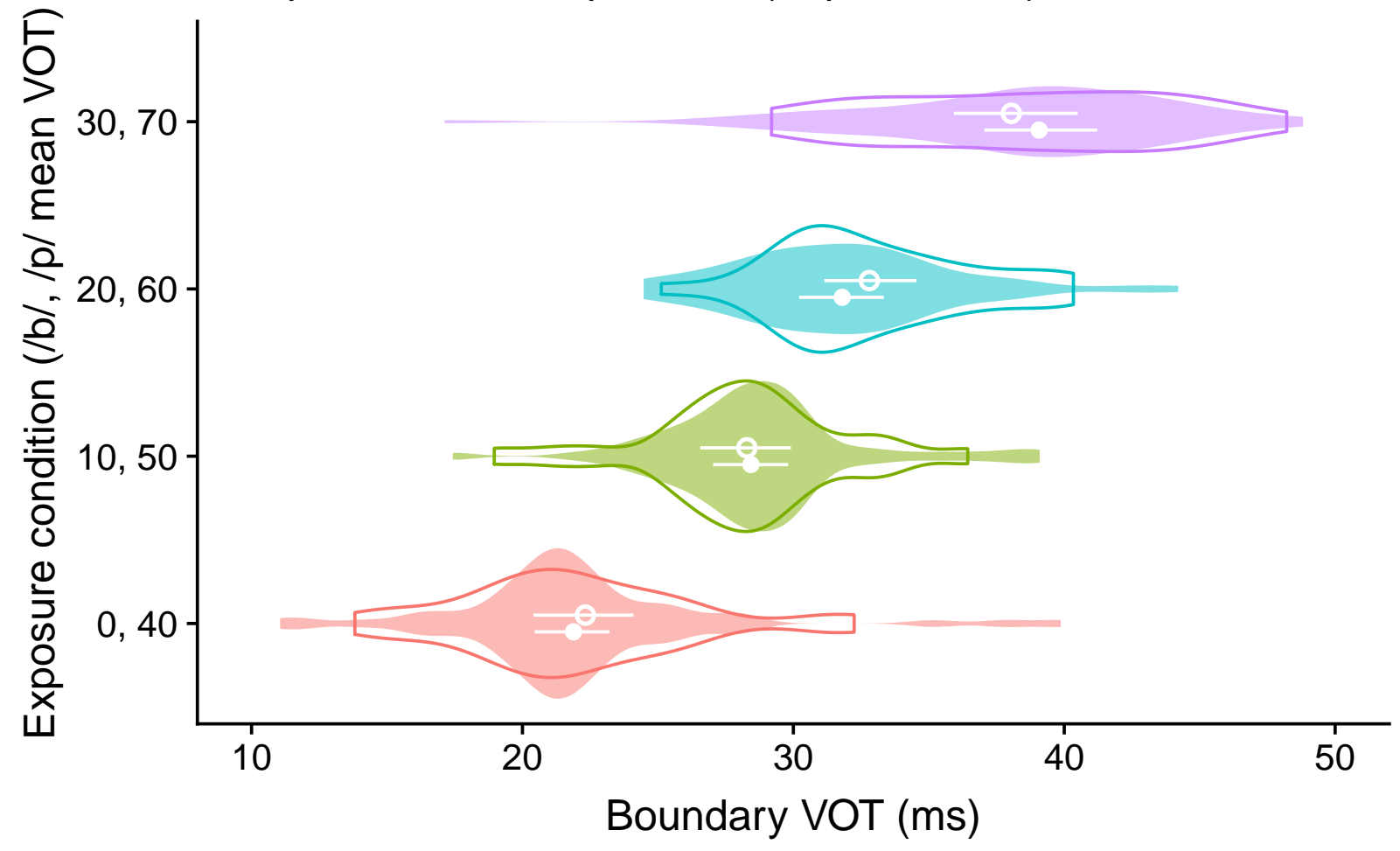

Figure 7: Providing 50\% labeled trials during distributional learning makes no difference in listener's category boundaries (Experiment 2, solid voilins/points) compared to purely unsupervised learning (Experiment 1, outlined violins/points, re-plotted from fig. 4). For ease of comparison with results from Experiment 1, the same axis limits are used as fig. 4; this lead to exclusion from the figure of five listeners with boundaries greater than $50 \mathrm{~ms}$ ( 2 in the $20 \mathrm{~ms} / \mathrm{b} /$ condition, 3 in the $30 \mathrm{~ms} / \mathrm{b} /$ condition). All participants are included in the regression model from which the fixed effects boundaries are estimated (points/lines). 
generative model.

One possible interpretation of this result is that the way that label information was provided made the labels somehow unavailable to update listeners' beliefs about how phonetic categories are acoustically realized. This is not particularly plausible, for two reasons. The first is that a wide variety of labeling information has been shown to be effective in phonetic recalibration, including lexical (Kraljic \& Samuel, 2005; Norris et al., 2003), visual/articulatory (Bertelson et al., 2003), and orthographic (Keetels, Schakel, Bonte, \& Vroomen, 2016) labels. The second is that listeners were highly sensitive to the labeling information in generating their responses on labeled trials, it was only on unlabeled trials where the presence of labels on other trials seems to make no difference.

Another possibility is that even without labels, listeners are able to pick up on the distributions they are exposed to, and so labeling some of the trials as a clue to which distribution they come from doesn't provide any additional information. If this is the case, how can we explain the discrepancy between the boundaries implied by those distributions and the boundaries listeners actually learned? Experiment 3 addresses this question via computational modeling.

\section{Experiment 3-Computational modeling}

Experiments 1 and 2 together present a puzzle: on the one hand, distributional learning appears to be "incomplete", with listeners using category boundaries that are reliably different from the boundaries implied by the VOT distributions they were exposed to. On the other hand, providing listeners extra information - in the form of category labels for half the VOTs they heard - did not have any effect on distributional learning. This suggests that listeners know the distributions that the exposure talker produces, but they just don't believe them, and hedge their bets by erring in the direction of a typical talker's boundary. Circumstantial evidence to this effect comes from the fact that the category boundaries that listeners do use appear to be a compromise between the category boundaries derived from the VOT distributions for the exposure talker and those produced by a typical talker of American English.

In this experiment I use a computational model to explore this possibility in more detail. Specifically, I use a model inspired by the ideal adapter framework (Kleinschmidt \& Jaeger, 2015), which is based on the idea that listeners maintain uncertain beliefs about the distribution of cues corresponding to each phonetic category, and update this beliefs incrementally based on their recent experience. Crucially, according to the ideal adapter, when listeners encounter an unfamiliar talker, they initially entertain a set of prior beliefs about the range of different phonetic cue distributions that an unfamiliar talker is likely to produce, which takes into account the variability across talkers that they have previously experience, and any available socio-indexical information that may be informative about this talker's cue distributions (Kleinschmidt, 2019; Kleinschmidt \& Jaeger, 2015). After direct experience with a talker, a listener will update their beliefs about that talker's cue distributions, bringing them into better alignment with the cue distributions that the talker has actually produced.

Why is this type of model a plausible explanation of the results of Experiments 1 and 2? First, this same type of model captures the changes in phonetic classification that happen during recalibration and selective adaptation (Kleinschmidt \& Jaeger, 2015) as well as during distributional learning due to different VOT distribution variances (Theodore \& Monto, 2019).

Second, the process of belief updating means that at any given point a listener's beliefs about that talker's cue distributions will be a compromise between what they expected a priori, and the distributions they have actually encountered. When a listener has limited experience with a particular talker, or especially strong, specific prior expectations, this compromise will be especially obvious because their prior beliefs will be more informative relative to their recent, direct experience with the talker.

Finally, one prediction of the ideal adapter is that listeners' prior expectations should be calibrated to the level (and type) of talker variability they have actually encountered (Kleinschmidt \& Jaeger, 2015). In this framework, the prior beliefs that listeners start from are based on listeners' expectations, on the one hand, about what a typical talker will produce, and on the other hand, how consistent talkers are in their realization of the given phonetic cue distributions. In the case of VOT distributions for word-initial stop voicing, there is relatively little variability across talkers in VOT distributions (Kleinschmidt, 2019), the distributional learning experiments here are exactly the kind of situations where listeners might be expected 
to bring strong prior expectations and hence show a clear compromise between those prior expectations and the distributions that they encounter in the experiments.

\subsection{Methods}

I use a Bayesian belief updating model, where listeners begin the experiment with a shared set of prior beliefs about the mean and variance of the VOT distributions for $/ \mathrm{b} /$ and $/ \mathrm{p} /$, and update these beliefs according to Bayes rule based on the VOTs they hear in the experiment. ${ }^{8}$

Specifically, a listener's uncertain beliefs about the mean and variance of each category $(/ \mathrm{b} / \mathrm{and} / \mathrm{p} /)$ are represented as Normal-Inverse Chi squared distribution. At the beginning of the experiment, every listener has the same prior beliefs, expressed in the model as parameters for the expected mean and variance ( $\mu_{0}$ and $\sigma_{0}^{2}$ ), and for their confidence in these beliefs ( $\kappa_{0}$ for the mean and $\nu_{0}$ for the variance). These confidence parameters are "pseudocounts", in that they are equivalent to assuming that the listener's expectations about the category mean is based on $\kappa_{0}$ prior observations from that category, and likewise for the variance and $\nu_{0}$. This prior distribution is a conjugate prior because after observing $n$ data points with sample mean $\bar{x}$ and variance $s^{2}$, the posterior distribution is also a Normal-Inverse Chi squared, with updated values of the four parameters:

$$
\begin{aligned}
\kappa_{n} & =\kappa_{0}+n \\
\nu_{n} & =\nu_{0}+n \\
\mu_{n} & =\frac{\kappa_{0} \mu_{0}+n \bar{x}}{\kappa_{0}+n} \\
\sigma_{n}^{2} & =\frac{1}{\nu_{n}}\left(\nu_{0} \sigma_{0}^{2}+n s^{2}+\frac{n \kappa_{0}}{\kappa_{0}+n}\left(\mu_{0}-\bar{x}\right)^{2}\right)
\end{aligned}
$$

These parameter updates have an intuitive interpretation (Kleinschmidt \& Jaeger, 2015): the confidence parameters are increased by the number of observations $n$ (which is why they are called "pseudocounts"), while the new expected mean and variance are weighted averages of the prior expectations and the observed values, with the weights determined by the pseudocounts and actual count, respectively. ${ }^{9}$

Given updated beliefs about the mean and variance of each category the probability of any particular VOT $x$ being /b/ vs. /p/ can be calculated by the marginal likelihood of $x$ under the /b/ and /p/ distributions (see Kleinschmidt \& Jaeger, 2015, for more details). The marginal likelihood is essentially a weighted average of the likelihood under all possible means and variance for each category, weighted by how probable each mean/variance is based on the VOTs observed from each category so far and the prior beliefs. These marginal likelihoods are then converted to a probability of responding /p/ or /b/ using Bayes' rule.

\subsubsection{Assumptions}

In actually implementing this model, I make a number of simplifying assumptions for the sake of tractability. First, and most importantly, I provide the model with the observed mean and standard deviations of the two exposure VOT distributions ( $\bar{x}$ and $s$ above). This substantially simplifies the complexity of the learning problem: in order to model unsupervised learning, a Bayesian model needs to average predicted behavior over distributional learning given each possible way that all the tokens could be jointly classified, weighted by the probability of that classification. In an experiment with 200 trials, each of which could be either /b/ or $/ \mathrm{p} /$, this amounts to more than $10^{60}$ possibilities, an impossibly large number to consider. There are approximations for unsupervised or semi-supervised belief updating, but they substantially increase the computational complexity and exploring them is left for future work.

Second, the use of a conjugate Normal Inverse Chi-squared prior is also an important simplification, as it means that the belief updating can be computed analytically. Combined with the first assumption,

\footnotetext{
${ }^{8}$ Note that, as in Kleinschmidt and Jaeger (2015), this model is an instantiation of only a component of the ideal adapter theory - the process of evidence accumulation within a single context - and does not stand in for the entire theory.

${ }^{9}$ For the variance update, there is an additional term that captures the possibility that a mismatch between the expected and observed mean could be due to a higher than expected variance.
} 
this means that the updated beliefs can be computed directly from the prior parameters and the observed count, mean, and variance of each category, rather than requiring an additional level of MCMC sampling to approximate.

\subsubsection{Model fitting procedure}

The free parameters of this model were the prior expected mean and variance of $/ \mathrm{b} / \mathrm{and} / \mathrm{p} /$, and the mean and variance prior pseudocounts (which were assumed to be the same for $/ \mathrm{b} /$ and $/ \mathrm{p} /$ because pilot simulations suggested it was not possible to reliably identify both). I also included a guessing rate parameter, which captured the fact that many listeners never reach floor or ceiling even for unambiguous high/low VOTs, and thus may be guessing on a proportion of trials.

The model was fit to data from Experiment 1 (unsupervised distributional learning) with the beliefupdatr package, which includes a Stan (Carpenter et al., 2017) implementation of this belief updating model and a convenient $\mathrm{R}$ ( $\mathrm{R}$ Core Team, 2020) interface. The trials were divided up into six blocks of equal length (37 trials). Responses in each block in each distribution condition were predicted based on updated beliefs at the halfway point of the block, using the sufficient statistics of that distribution condition.

No "random effects" (hierarchical effects by subject) were included. This is a difficult choice to make. On the one hand, there likely are differences in listeners' prior expectations and learning rates, and ignoring these ignores important variability in the data. On the other hand, including such random effects by subjects introduces many additional degrees of freedom into the model, and increases the number of data patterns it could fit. This is especially dangerous in this particular case since each listener only encountered a single set of distributions, and so random effects for subjects effectively introduces unconstrained flexibility in how the model captures differences between distributions. So, I opted not to include random effects in the belief updating model, so as to make as rigorous as possible a test of its ability to capture differences in distributional learning found above. However, the data and analysis scripts are available online for the interested reader to explore the consequences of alternative choices.

\subsubsection{Model evaluation}

The goodness-of-fit is evaluated using the PSIS-LOO method of Vehtari, Gelman, and Gabry (2017). This method approximates leave-one-out cross-validation to provide an estimate of the out-of-sample prediction error from the posterior samples of the model. This provides a goodness-of-fit measurement that penalizes overly complex models in a way that is directly (approximately) related to their inability to generalize beyond the data they were fit to, and as such is a more robust measure for comparing between different models than indirect metrics like AIC or BIC which rely on counting numbers of parameters or WAIC/DIC which are hard to estimate reliably from posterior samples (see Vehtari et al., 2017, for more discussion). Because data was nested within subject, I used subjects as the cross-validation units (instead of individual trial responses). I report the expected log-posterior density in the form of LOOIC, which is on the deviance scale $(-2$ times the log-likelihood) like AIC. On this scale, lower is better, corresponding to higher predictive log likelihood (better-fitting or more parsimonious models). Finally, because these are estimated from a finite dataset, there is uncertainty associated with them (and the differences between models), which is indicated by the standard error of the estimate (or difference).

The belief updating model was compared with two other models via LOOIC (both fit via brms, Bürkner, 2017). The first was a "null model" with a constant probability of responding /p/. The second was a baseline model similar to the logistic regression model used to analyze the data above, with two differences to make it a suitable baseline. First, because the belief-updating model fit all subjects with a single set of prior beliefs, the baseline model included no random effect, while maintaining the fixed effects structure (condition, trial, VOT, and their interactions). Second, because the belief-updating model included a "guessing rate" parameter that varied over the experiment, the baseline model also included a variable guessing rate (via a brms mixture family with varying weight). As in the belief updating model, the guessing rate was allowed to vary freely over the six blocks of 37 trials. 


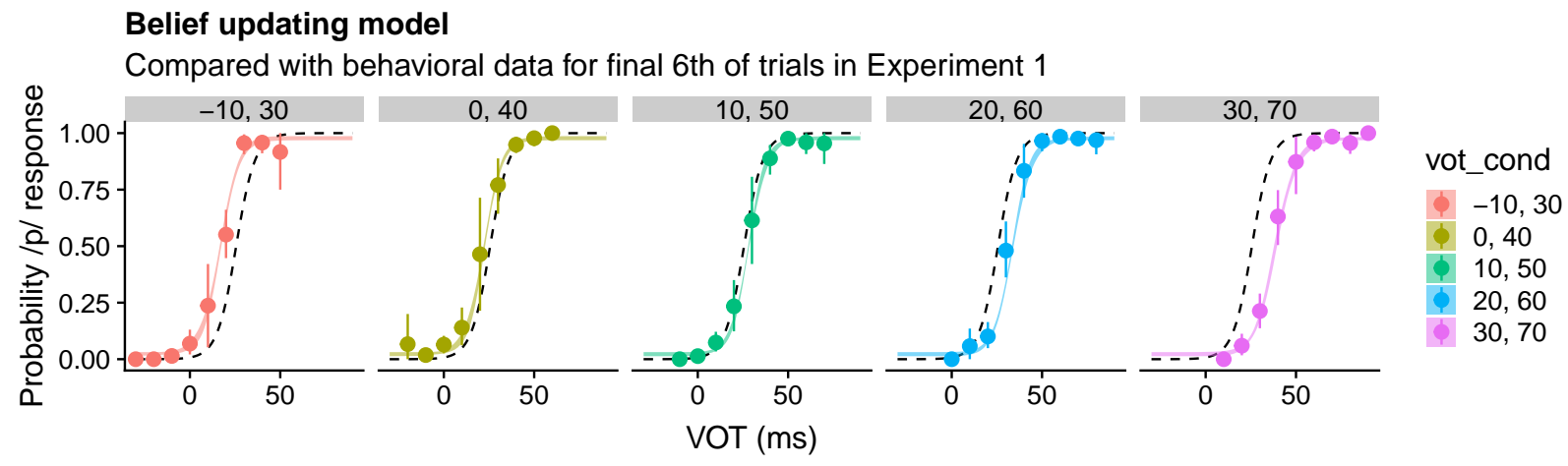

Figure 8: Belief updating model classification functions (thin ribbons) vs. average probability /p/ response from Experiment 1, both during the final sixth of trials. Points show the average proportion of $/ \mathrm{p} /$ responses, and CIs show $95 \%$ bootstrapped CIs over subjects. Ribbons show $95 \%$ Bayesian credible interval for model posterior predictions, and the dashed black lines show classification function from the inferred prior.

\subsection{Results}

The belief updating model can be evaluated in a number of ways. At the highest level, it quantitatively fits the data well, as shown by Table 1. The LOOIC for the model is better (lower) than the LOOIC of two baseline models: conclusively so for the null, intercept-only model, and numerically better for the logistic regression baseline, although the $\mathrm{SE}$ of that difference is large so it is not possible to say conclusively. ${ }^{10}$ Whether or not the difference is conclusive, the belief updating model fits the data as well as or better than the logistic regression baseline despite having many fewer free parameters (excepting the common lapse rate parameters, 6: prior mean and variance for two categories, and prior confidence in mean and variance; versus 20: a separate bias and VOT slope for each of five conditions, plus linear change in these with trial).

Next I will show three ways of evaluating the model's fit qualitatively. First, I discuss the outcome of distributional learning predicted by the model: does the model capture the classification functions that listeners use at the end of the experiment? Second, I look at the time course of the learning that the model takes: does it capture the how classification functions change with increasing exposure to the experimental distributions? Third, and finally, I will examine whether the shared prior beliefs - the starting point for belief updating in the model - match the distributions produced by a typical talker of American English.

Table 1: LOOIC values for the belief updating model and two baselines (lapsing logistic regression and a null, intercept-only model). The individual LOOIC values are shown as well as the difference between the belief updating model and the others, along with the standard errors of the estimates associated with each.

\begin{tabular}{lrrrr}
\hline & LOOIC & (SE) & LOOIC diff. & (SE) \\
\hline Belief updating & 17,485 & 689 & & \\
Baseline (logistic) & 17,502 & 697 & 17 & 975 \\
Null (intercept-only) & 42,347 & 74 & 24,862 & 689 \\
\hline
\end{tabular}

\footnotetext{
${ }^{10}$ Although note that any difference - especially one on the order observed here - using analogous model-comparison statistics like AIC, BIC, etc. is traditionally treated as evidence in favor of the lower scoring model, despite the fact that all of these scores are uncertain estimates based on a finite set of data. LOOIC is in this way a more honest measure in that it makes it straightforward to get an approximate measure of how stable this estimate is.
} 


\section{Model predicted and actual learning curves Change in $/ \mathrm{b} /-/ \mathrm{p} /$ category boundary with learning}

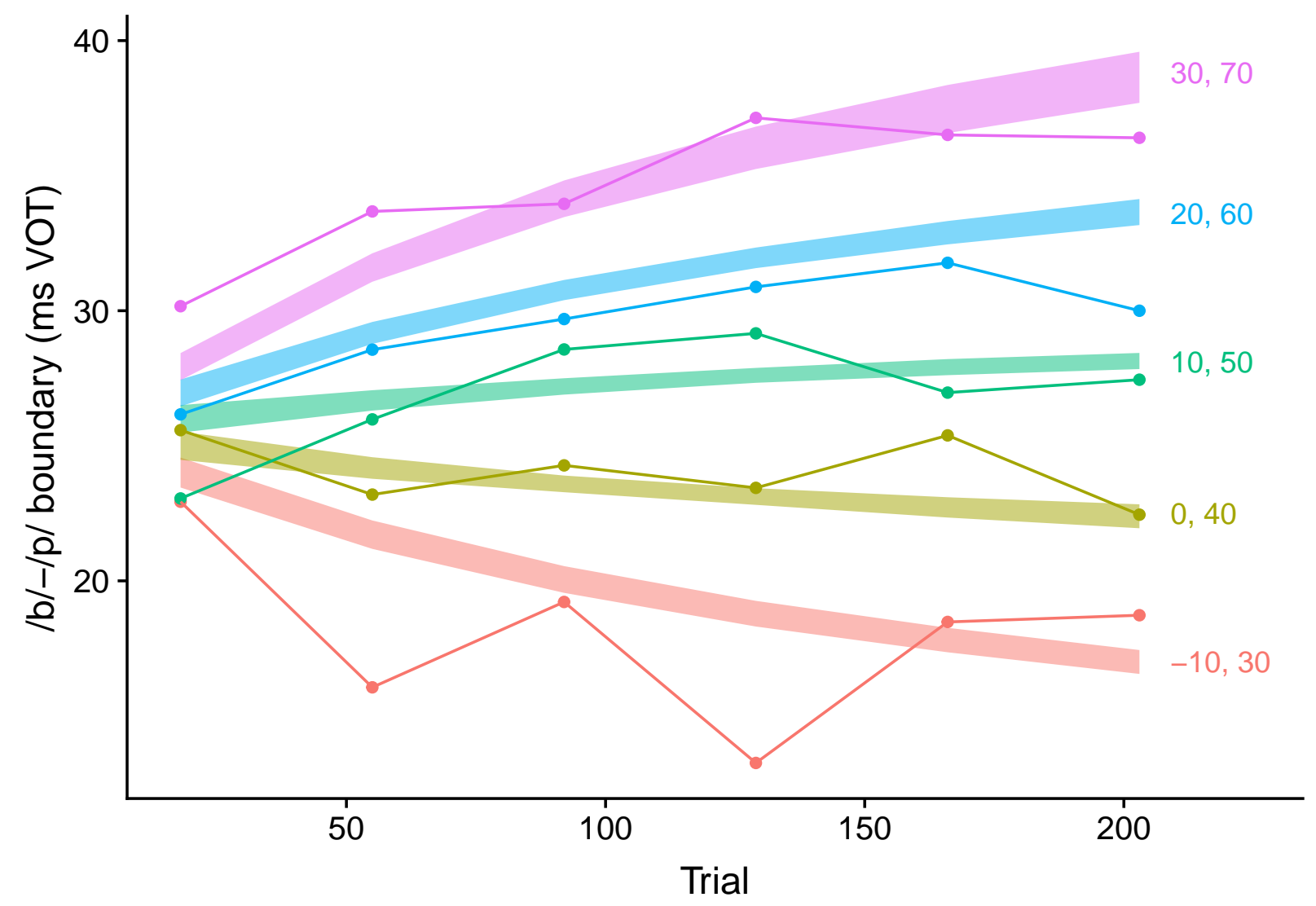

Figure 9: Belief updating model's learning curves (ribbons, $95 \%$ credible intervals) compared with empirical category boundary, defined as the VOT where subjects' responses (linearly interpolated) are 50\% /p/, 50\% /b/. Both model and empirical boundaries are estimated for each sixth of trials (blocks of 37 out of 222).

\subsubsection{Outcome of learning}

First, does the belief updating model successfully capture the overall pattern of incomplete distributional learning by the end of the experiment? This serves as a check whether the overall pattern of distributional learning is compatible with an "ideal adapter" belief updating process, where every listener starts with the same, shared prior expectations about an unfamiliar talker's VOT distributions for $/ \mathrm{b} /$ and $/ \mathrm{p} /$.

Figure 8 shows the belief-updating model's predicted classification functions in the final sixth of trials (186-222) in the experiment, along with the average probability of a / $/$ / response for each VOT (plus bootstrapped $95 \%$ CI over listeners). Qualitatively, the model provides a good fit to the overall classification functions for each VOT condition, and the estimated boundaries align well with the observed boundaries in each case. This suggests that the pattern of incomplete distributional learning found in Experiment 1 is compatible with a belief updating model. This is not terribly surprising, since the category boundaries listeners were using by the end of the experiment appeared to reflect a compromise between a typical talker and the experimental talker's distributions, and a Bayesian belief-updating model formalizes exactly this kind of compromise in a statistical framework. However, the ability of the model to quantitatively capture the pattern of distributional learning with a single set of starting beliefs serves as an existence proof, putting this explanation of the constrained distributional learning observed above in Experiment 1 on firmer footing. 


\subsubsection{Learning curves}

Next, how well does the belief-updating model capture the learning curve, or change in category boundaries with further experience? Figure 9 shows the belief-updating predicted learning curves, compared with the empirical boundaries (points/lines) estimated for each condition in each sixth of the experiment (blocks of 37 trials). The belief updating model qualitatively captures the change in category boundaries, both within each condition and across conditions. Boundaries start off quite similar at the beginning of the experiment, and gradually diverge as listeners gain more exposure to the particular VOT distributions of their condition.

Comparing these modeled learning curves with the behavioral data directly is not straightforward. Figure 9 shows one estimate of the changing category boundaries, estimated directly from the data in each sixth of the experiment (e.g., the VOT where the average proportion-/p/ response curve crosses $50 \%$ in each condition). Other reasonable possibilities include a boundary calculated from the regression model reported in Experiment 1 (which includes a linear change in intercept, or $/ \mathrm{p} /$ bias, over trials, as well as a linear change in the slope of the VOT boundary), or a more complex regression model using a spline term in each condition to capture non-linear changes in the offset (but not the slope).

All of these methods agree qualitatively on where the boundaries are later in the experiment, when listeners make very few errors or random responses. Where they disagree (with each other, and with the belief updating model) is in the estimated boundaries for the early trials, where listeners make many random or guessing responses, even on unambiguous VOTs. In the belief updating model, this is captured by the "lapse rate" or the proportion of trials on which the simulated subjects simply guess randomly, which is allowed to vary over trials (Figure 10). The inferred lapse rate is nearly $12 \%$ in the first sixth of trials (1-37), while for the remainder of the experiment it is closer to $5 \%$. Models that don't take into such guessing into account are subject to mis-estimate the boundary (location and slope Clayards et al., 2008; Wichmann \& Hill, 2001), and so for this reason I chose the more interpretable - if somewhat noisier - direct estimate of the boundary over the estimates from either of the regression models for comparison with the belief updating model.

\subsubsection{Inferred prior beliefs}

Finally, what are the prior beliefs that the model infers listeners are bringing to this task? There are two relevant aspects of the inferred prior beliefs: the content of those beliefs, and their strength. The strength corresponds to how confident the modeled listener is in their prior beliefs, which is represented in the model in the prior confidence pseudocounts for the means $\left(\kappa_{0}\right)$ and variance $\left(\nu_{0}\right)$. These parameters correspond to the number of observations required to get to a point where the listener's updated beliefs are in equal parts informed by their prior expectations and recent experience.

Based on the data from Experiment 1, listeners are highly confident in their prior beliefs. The expected prior pseudocount for the means was $\kappa_{0}=160$ (95\% credible interval of $\left.[75,780]\right)$. This is nearly twice the number of trials in the experiment from each cluster (111 each, for a total of 222), meaning that by the end of the experiment the model's beliefs about the mean VOTs for $/ \mathrm{b} /$ and $/ \mathrm{p} /$ are still primarily determined by the prior beliefs. Similarly, the expected prior pseudocount for the variances $\nu_{0}=510(95 \%$ credible interval of $[160,1000])$, which is much larger than the number of trials from each cluster.

Together, these parameters suggest that listeners have strong prior beliefs about the VOT distributions associated with $/ \mathrm{b} /$ and $/ \mathrm{p} /$, and moreover that their beliefs in the variance of VOTs is stronger than their beliefs in the means. In turn, this suggests that the distributional learning observed in Experiment 1 is driven mostly by changes in listeners' beliefs about the underlying means, with only small contributions from changes in the variance (e.g., a relaxation in their standards for what counts as a good $/ \mathrm{b} / \mathrm{or} / \mathrm{p} /$ ).

However, as in previous work with similar belief updating models (Kleinschmidt \& Jaeger, 2015), the posterior distribution of these parameters was bimodal. One mode that puts more confidence in the means $\left(\kappa_{0}>\nu_{0}\right)$ and adapts mostly by updating beliefs in the variance, while the other puts more in the variances $\left(\nu_{0}>\kappa_{0}\right)$ and adapts by shifting. Nevertheless, the "shifting" solution is preferred, with $\nu_{0}>\kappa_{0}$ in the majority of MCMC samples (Bayesian $p$ value of 0.85 ).

Figure 12 shows the prior expected VOT distributions for $/ \mathrm{b} /$ and $/ \mathrm{p} /$ that are most likely given the distributional learning behavior (maximum a posteriori/MAP), along with . The belief updating model recovers distributions that are similar but not identical to those estimated by Kronrod et al. (2016) (based on classification and discrimination data, assuming that /b/ has a mean VOT of $0 \mathrm{~ms}$ ). The inferred 


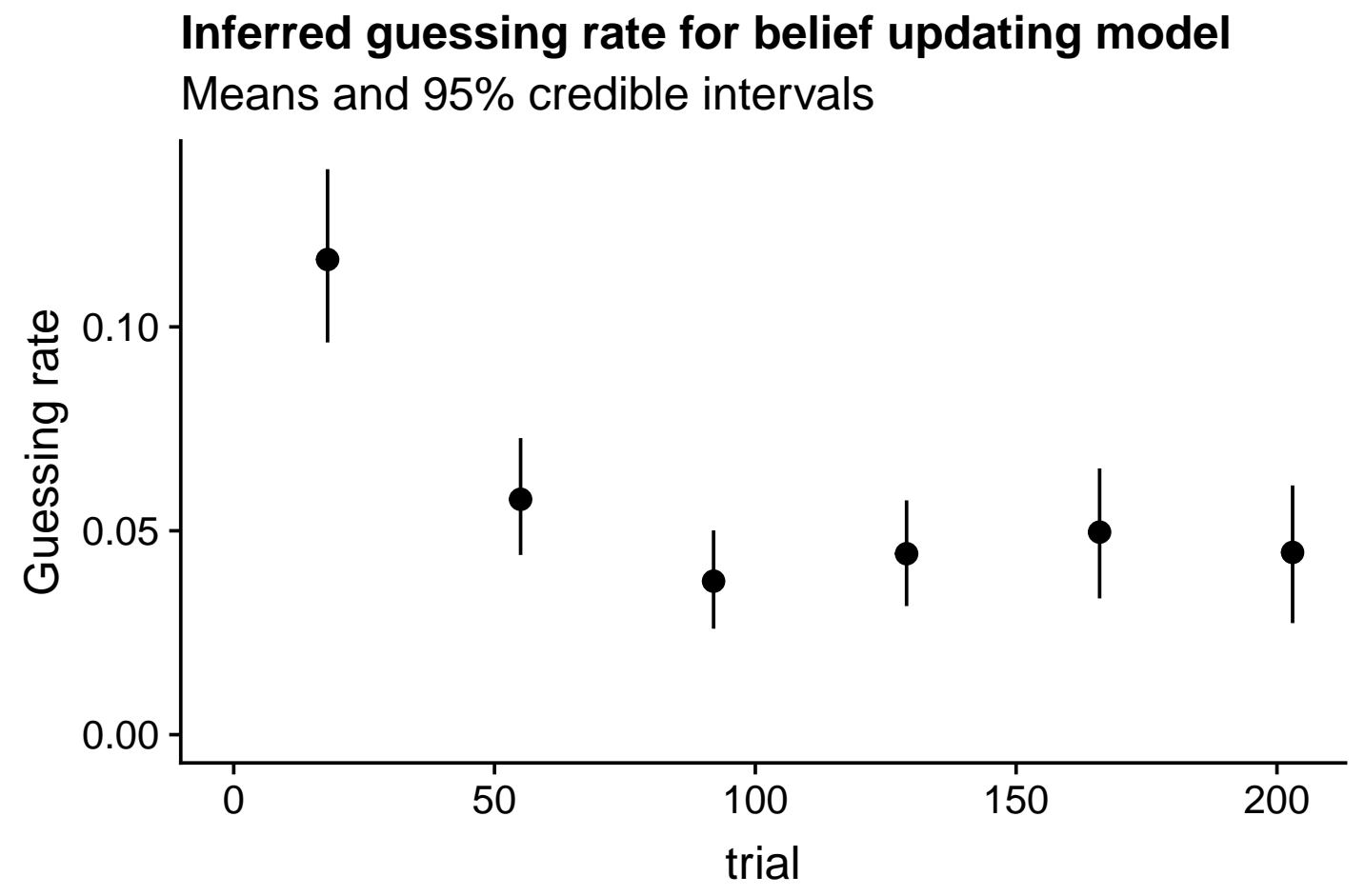

Figure 10: Lapse (guess) rate inferred in the belief updating model for each 1/6th of trials in Experiment 1. The inferred lapse rate starts rather high (nearly $12 \%$ ) but decreases to around $5 \%$ after the first $1 / 6$ of trials $(1-37)$ 


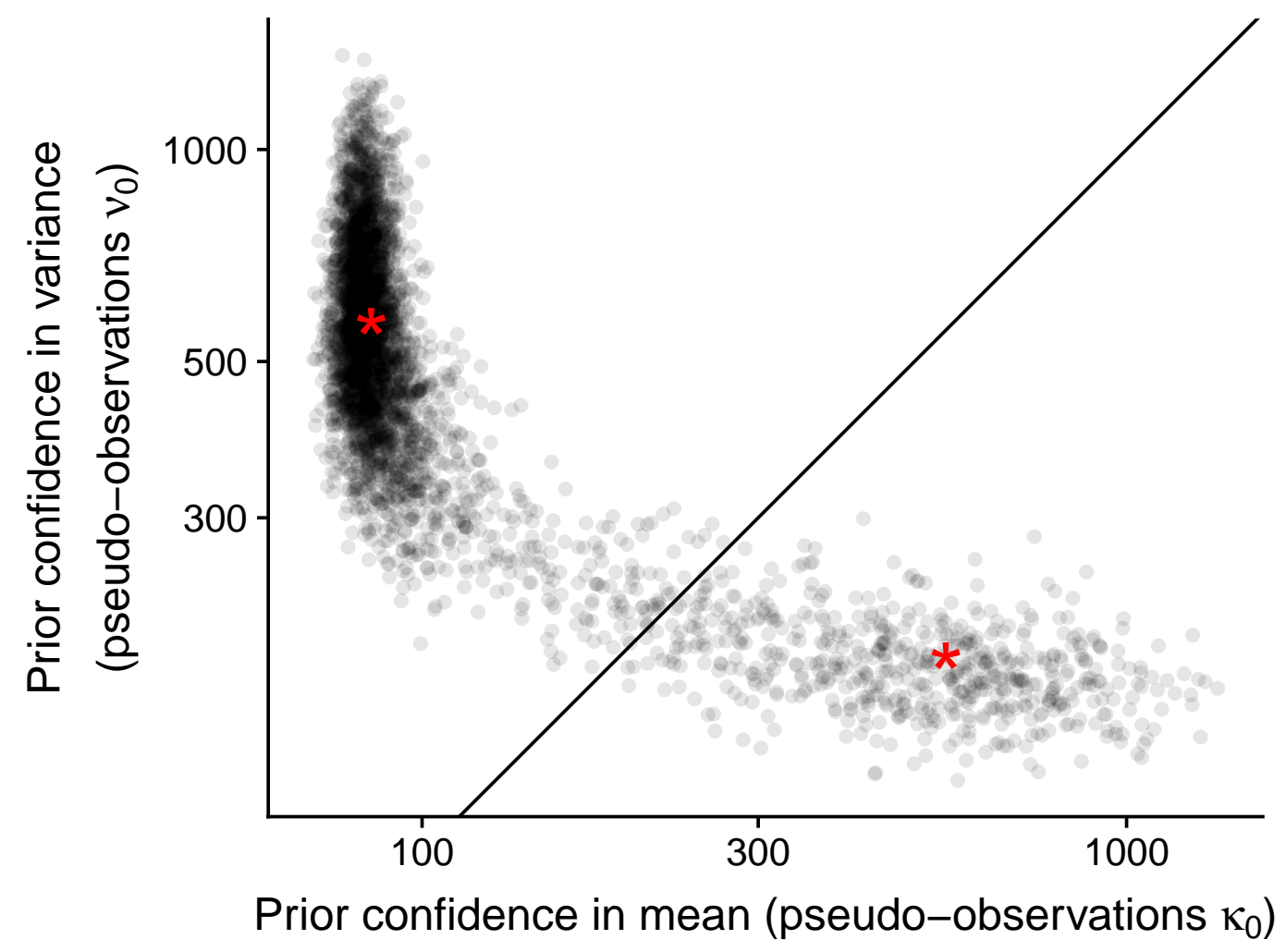

Figure 11: Sampled values of prior confidence parameters from belief updating model fit to Experiment 1. Upper left shows values where $\kappa_{0}<\nu_{0}$ ("shifting"), and lower right shows values where $\nu_{0}<\kappa_{0}$ ("expanding"). Red stars show approximate MAP values for each kind of solution (individual sample with the highest posterior). 


\section{Inferred prior beliefs}

Maximum a posteriori beliefs and 100 samples from belief updating model

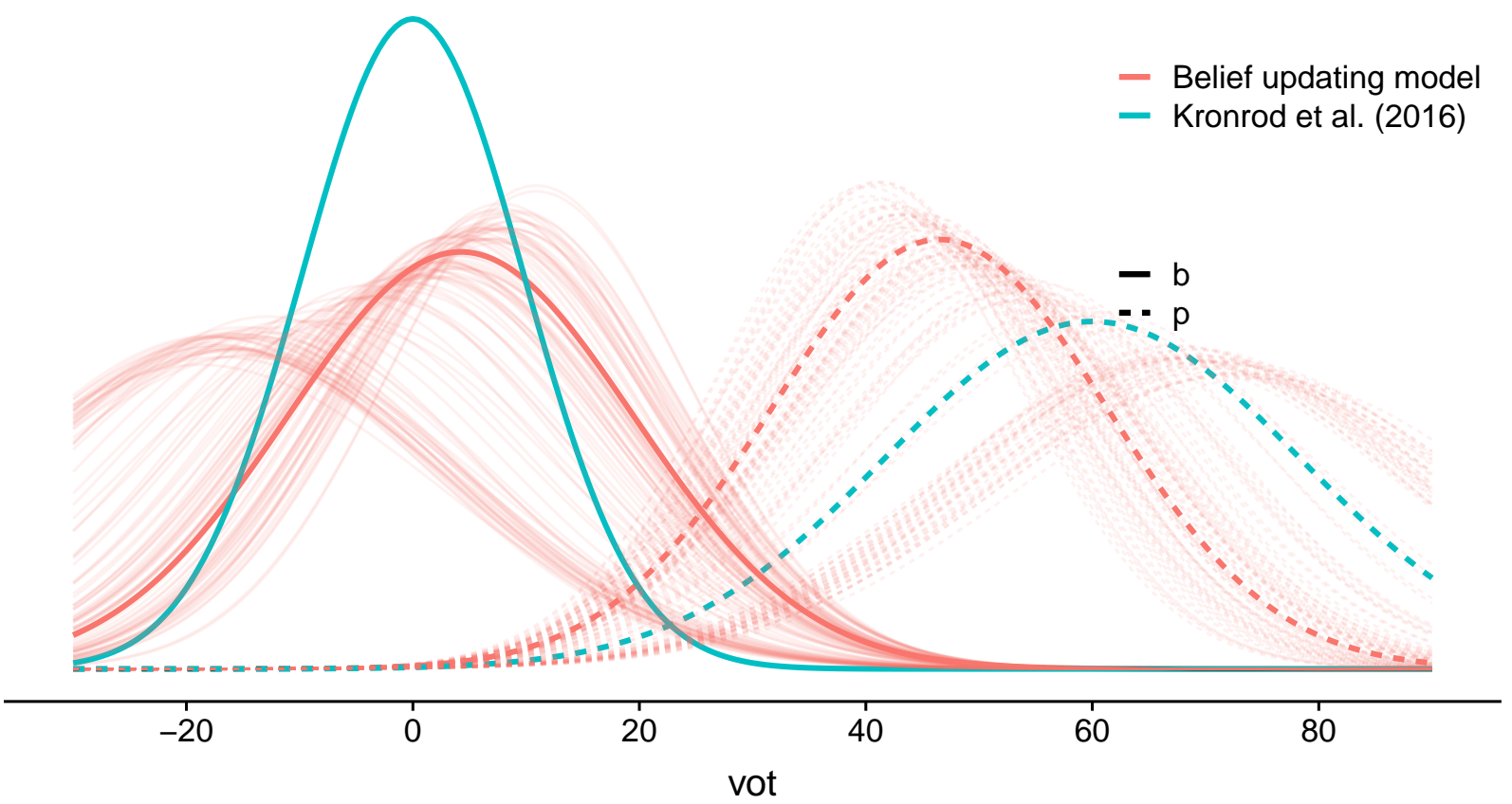

Figure 12: Inferred prior beliefs from belief updating model, compared with VOT distributions estimated by Kronrod, Coppess, and Feldman (2016). Inferred priors are derived using the maximum a posteriori (MAP) estimate of the expected prior mean and variance parameters $\mu_{0}, \sigma_{0}^{2}$ (heavy lines). To give a sense of the range of uncertainty that the model has about listener's prior beliefs based on their behavior in the experiment, also plotted are the prior beliefs from 100 values sampled from the posterior (light lines). 
distribution for $/ \mathrm{p} /$ has a slightly lower mean but very similar variance, while $/ \mathrm{b} /$ has a similar mean but higher variance. Notably, the variance of the two distributions is quite similar, and the difference between the prior means for $/ \mathrm{b} /$ and $/ \mathrm{p} /$ is very close to $40 \mathrm{~ms}$, the difference between the cluster means of the exposure distributions.

Also shown in Figure 12 are 100 samples from the distribution of prior beliefs given listeners' behavior in Experiment 1. This shows that the behavior does not uniquely constrain these prior beliefs, and is instead compatible with belief updating starting from prior beliefs with a range of means and variances.

\subsection{Discussion}

The results of the belief updating modeling show that the pattern of constrained distributional learning from Experiment 1 can be explained as belief updating starting from a shared set of prior beliefs. Both the outcome of this learning and the learning curves are captured by a Bayesian belief updating model modeled on previous work on phonetic recalibration (Kleinschmidt \& Jaeger, 2015). This suggests that despite their superficial differences, both recalibration and distributional learning may be reflections of the same underlying process of belief updating (see also Theodore \& Monto, 2019).

\subsubsection{Content of prior beliefs}

The starting point for belief updating that is most consistent with the distributional learning behavior from Experiment 1 is reasonably similar to VOT distributions produced by a typical talker of American English. The notable exception to this is that the belief updating model infers that the $/ \mathrm{b} /$ and $/ \mathrm{p} /$ distributions have similar variance, whereas in actual speech the /b/ distribution has much lower variance compared with $/ \mathrm{p} /$. This likely reflects the fact that such variance asymmetry is hard to detect from purely classification data. Kronrod et al. (2016) used discrimination data in addition to classification data in order to estimate listeners' beliefs about the underlying variances of voiced and voiceless stops (in addition to the sensory uncertainty associated with VOT itself).

\subsubsection{Strength of prior beliefs}

The belief updating model also suggests that these prior beliefs are relatively strong, with listeners acting as if their prior beliefs were based on $2-5 \times$ more VOT samples prior than they actually encounter during the experiment itself. This is in stark contrast to the results of the same belief updating model applied to /b/-/d/ recalibration (Kleinschmidt \& Jaeger, 2015), which found that listeners have relatively weak prior beliefs. Despite the fact that the number of trials in the experiments were similar (about 200), Kleinschmidt and Jaeger (2015) found that recalibration of /b/-/d/ was best explained by prior beliefs that were 10 times weaker than here (prior pseudocounts on the order of 10s of observations, rather than 100s like the current results). Why this discrepancy? One possibility is the different learning tasks: in a recalibration tasks like that being modeled by Kleinschmidt and Jaeger (2015), listeners repeatedly hear stimuli chosen to be acoustically ambiguous (between /aba/ and /ada/ in this case), paired with a label of some kind of label (like a visual cue to the articulatory gesture; Bertelson et al., 2003; Vroomen, van Linden, de Gelder, \& Bertelson, 2007). While my Experiment 2 found that providing labeling information did not appreciably change the speed or completeness of distributional learning, it's possible that by targeting ambiguous stimuli specifically - rather than the wider range of stimuli used here -could lead to more reliance on labeling information (although the fact that exposure to not-fully-ambiguous or even prototypical stimuli leads to equally rapid belief updating suggests this is unlikely; Kleinschmidt \& Jaeger, 2015).

Another possibility, proposed by Kleinschmidt and Jaeger (2015), is that the strength of listeners' prior beliefs about a particular cue distribution is related to how much that particular distribution varies across talkers: when talker variability is low, an ideal adapter will use strong prior beliefs and thus be relatively inflexible. While there is some talker variability in VOT distributions (Allen et al., 2003; Chodroff \& Wilson, 2017), there is much less variability than in vowel formant frequencies (Kleinschmidt, 2019). Steady-state vowel formant frequencies are not exactly the same as the formant trajectories which are the primary cue for distinguishing voiced intervocalic stops, but there is still likely to be similar levels of talker variability in such spectral cues. 


\section{Experiment 4}

The evidence presented thus far suggests that adult distributional learning is constrained in substantial ways (Experiment 1), that these constraints cannot be attributed to uncertainty about the intended category due to the minimal pair words used (Experiment 2), and that the observed constraints are at least consistent with belief updating starting from a shared set of prior beliefs (Experiment 3). One interpretation of the modeling results from Experiment 3 is that the prior beliefs that the model infers as the starting point for distributional learning actually correspond to the real contents of listener's prior beliefs. However, despite the fact that some of the VOT distributions tested thus far deviate substantially from a typical talker of American English, in reality they cover only a rather narrow range of possible VOT distributions.

In this final experiment, I test listeners' distributional learning from distributions that vary more dramatically, in two ways. First, I include distributions with large negative VOTs. On the one hand, negative VOTs (prevoicing) are common cross-linguistically, and are also produced by many speakers of American English in word-initial voiced stops (Goldrick, Vaughn, \& Murphy, 2013; Lisker \& Abramson, 1964). On the other hand, word-initial voiced stops are canonically analyzed as un-aspirated un-voiced stops with VOTs around zero, and American English listeners have difficulty in recalibrating their classification boundaries to negatively-shifted VOT distributions (Sumner, 2011), so there may be less flexibility in how they are interpreted relative to positive VOTs.

Second, in Experiments 1 and 2 the means of the two VOT distributions vary in lock-step. There is some evidence from production data (Chodroff \& Wilson, 2017) that the means of different VOT distributions may be positively correlated across talkers in this way. If listeners are sensitive to this structured variation across talkers, it may result in additional constraints on distributional learning not detectable with the range of distributions tested in Experiments 1 and 2.

In both cases, the belief-updating model from Experiment 3 does not include these possible constraints. In the first case, it assumes that there are exactly and only two VOT distributions, and cannot account for the fact that in reality there are three clusters of VOT of word-initial stops in American English, with means that are negative (prevoiced), approximately zero (un-aspirated), and positive (aspirated). In the second case, the model assumes that the priors on the VOT distributions for $/ \mathrm{b} /$ and $/ \mathrm{p} /$ are independent, and thus by design cannot capture additional constraints on distributional learning that might arise from experience with correlated variability across different phonetic categories.

Thus, this final experiment provides a challenging test of the ability of the prior beliefs inferred from the pattern of distributional learning for various distributions in Experiment 1 to generalize to quite different situations.

\subsection{Methods}

As in Experiment 1, listeners performed a word identification task without labeling information, selecting from a pair of images corresponding to /b/-/p/ minimal pair words beak-peak, beach-peach, and bees-peas. Each listeners heard one of five different synthetic "accents," corresponding to a different bimodal distributions of VOT. Across these distributions, the mean VOT for /b/ and /p/ varied semi-independently (Figure 13). These distributions cover a wider range of implied category boundaries (from - 15 to $45 \mathrm{~ms}$ VOT) than Experiment 1 (10 to 50ms), especially on the lower end. They also varied in the distance between cluster means (from 40 to 130ms VOT), unlike Experiment 1 which maintained a constant separation between /b/ and $/ \mathrm{p} /$ mean VOT of $40 \mathrm{~ms}$. Note that the condition with /b/ mean of $10 \mathrm{~ms}$ and $/ \mathrm{p} /$ mean of $50 \mathrm{~ms}$ VOT is the same as in Experiment 1. This condition was included to provide a way of comparing with Experiment 1, since the design of Experiment 4 required a change in procedure (discussed next).

\subsubsection{Procedure: assessing category boundaries}

The procedure was identical to Experiment 1, with one exception. After completing the 222 trial exposure phase as in Experiment 1, listeners completed a test phase in order to assess their category boundaries. This phase consisted of 70 additional trials with VOTs evenly distributed from -10 to $50 \mathrm{~ms}$ in $10 \mathrm{~ms}$ steps $(10$ each). This additional phase was necessary because of the large separation between $/ \mathrm{b} /$ and $/ \mathrm{p} /$ clusters in the exposure distributions meant that in most conditions there were no trials with VOTs anywhere near the predicted (or typical) boundaries. 


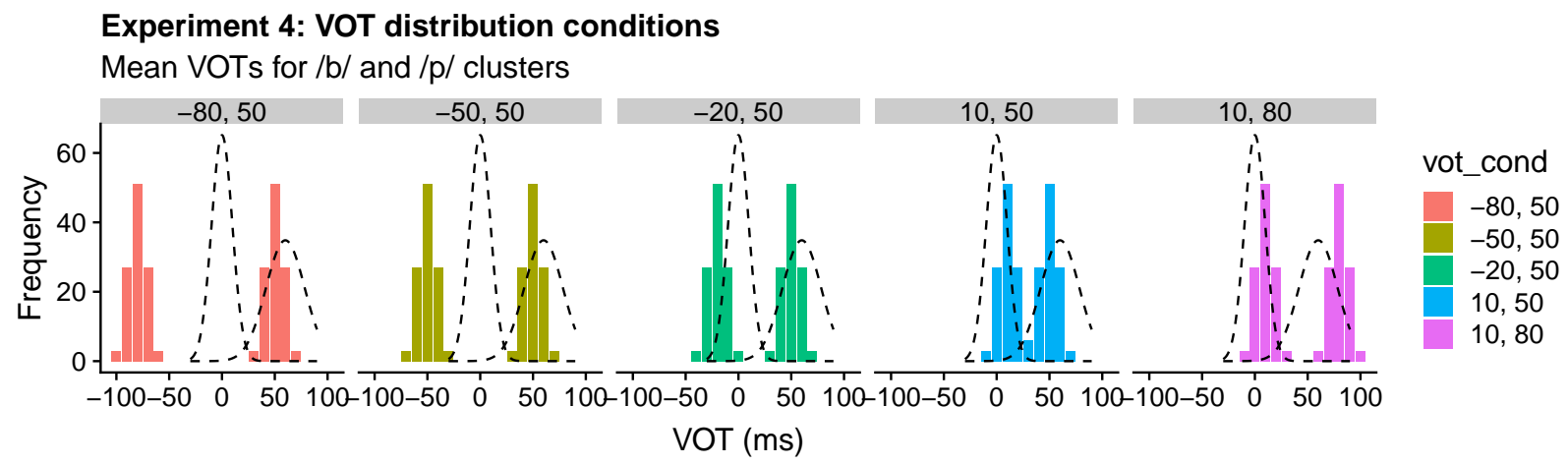

Figure 13: In Experiment 4, each subject heard a talker that produced one of these five VOT distributions. The variance of each category was constant across conditions and the same as in Experiments 1 and 2, but the means varied semi-independently. The first four conditions had a mean /p/ VOT of 50ms, with /b/ mean from -80 to $10 \mathrm{~ms}$ VOT. The $10 \mathrm{~ms} / \mathrm{b} /, 50 \mathrm{~ms} / \mathrm{p} /$ condition was the same exposure distributions as in Experiment 1. The final condition had means of $10 \mathrm{~ms}$ for $/ \mathrm{b} /$ and $80 \mathrm{~ms}$ for $/ \mathrm{p} /$. Distributions for a typical talker are shown in dashed lines.

Critically, listeners were not told about the change from exposure to test phase: the procedures were identical, and there was no break in between. Besides the change in VOT distributions, there was no way for listeners to tell that they had entered the test phase. Of course, if listeners are actually (as hypothesized) learning these distributions, their behavior may well change as they proceed through the test phase, gradually erasing any effect of the differences in the exposure distributions. Thus, when analyzing data from the test phase, whenever possible analyses are focused on the early parts of the test phase, when listeners behavior should be minimally affected by the change in distributions.

As in Experiment 1, listeners' adaptation was evaluated via their classification functions, estimated with a hierarchical Bayesian logistic regression model via brms (Bürkner, 2017). In addition to VOT, a predictor for trial was also included, in order to account for any (un-)learning effects that might happen during posttest, as well as all interactions between these and condition. ${ }^{11}$ In order to visualize the fitted classification functions and to estimate listeners' category boundaries, I used the classification function estimates from the trial halfway through the first third of the post-test. ${ }^{12}$

\subsubsection{Participants}

A total of 124 participants were recruited via Amazon's Mechanical Turk. Subjects were paid $\$ 2.50$ for participation. The task took subjects about 25 minutes to complete (because of the additional post-test, discussed above). As in Experiment 1, subjects were excluded who failed to classify words with unambiguous VOTs reliably during exposure $(n=5)$. After exclusion, 119 subjects remained for analysis (no subjects had previously participated in previous experiments). Subjects were evenly distributed across conditions (range of 23 to 25 per condition). 


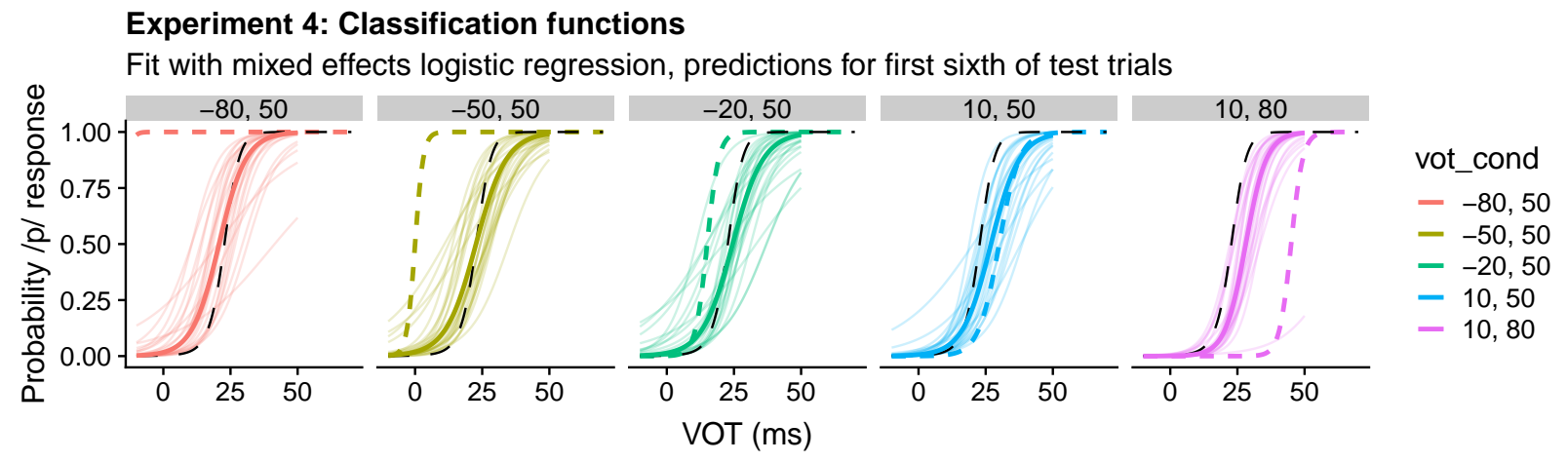

Figure 14: Classification functions from Experiment 4. Fine lines show individual subjects' classification functions (estimated based on random effects/subject-level predictors in the Bayesian mixed effects model) and thick solid lines show the group-level estimates (based on the fixed effects). Thick colored dashed lines show the ideal categorization function given the exposure distributions, and the thin black dashed lines show the categorization function for a typical talker.

\subsection{Results}

Figure 14 shows the classification functions produced by listeners during the first sixth of the test phase of Experiment 4 (estimated by Bayesian hierarchical logistic regression), and Figure 15 shows the corresponding estimates of the $/ \mathrm{b} /-/ \mathrm{p} /$ boundaries. First, consider the $10,50 \mathrm{~ms}$ condition. This condition serves as a replication of the condition with the same exposure distributions from Experiment 1, and a check of the posttest procedure used in the other conditions of Experiment 4 to measure listeners' category boundaries. As Figure 15 shows, listeners in the 10, 50ms condition from Experiment 4 produced very similar-if somewhat more variable - category boundaries in the post-test (filled violin) compared with the boundaries measured in the 10, 50ms condition Experiment 1 (empty violin), despite the fact that the Experiment 4 boundaries were measured during post-test and the Experiment 1 boundaries during exposure. There is also more uncertainty associated with the estimates of the group-level (fixed-effects) boundaries as well (the filled vs. open white circles and associated credible intervals), even though the estimates themselves are largely in agreement (27 vs. $28 \mathrm{~ms}$ VOT). On the one hand, this validates the use of the post-test procedure in Experiment 4 as a way of measuring the outcome of distributional learning. On the other hand, the greater variability in these measurements suggests that the use of a post-test might reduce the power to detect differences in learning between conditions of Experiment 4.

Next, exposure to large negative VOTs produces comparably small shifts in the /b/-/p/ category boundary. In fact, in the conditions with a lower mean of $-80,-50$, and $-20 \mathrm{~ms}$, listeners' category boundaries seem to cluster around the boundary for a typical talker, rather than lying in between the typical talker's boundary and the exposure talker's ideal boundary as in Experiment 1. Moreover, while the differences in boundaries between these three conditions are trending in the expected direction, with more negative VOTs expected to lead to lower category boundaries, these differences are small (on the order of 0-5ms, compared with exposure distributions with $15 \mathrm{~ms}$ differences between implied boundaries) and not particularly reliable (going in the expected direction with only $p=0.8^{13}$ for the $-20 \mathrm{~ms}$ vs. $-50 \mathrm{~ms} / \mathrm{b} /$ conditions and $p=0.85$ for

\footnotetext{
${ }^{11}$ The complete formula for the model was respP $\sim 1+\operatorname{vot} \_$cond $*$ vot_s $*$ trial_s $+(1+$ vot_s $\mid$ subject $)$. As before, VOT and trial were centered and scaled to have zero mean and unit variance. Because the regression coefficients were not directly interpreted (the fitted category boundaries used instead), the default (treatment) coding was used for condition. The default priors for parameters were used.

${ }^{12}$ The predictions from a regression model are more uncertain and more driven by noise at the extreme ranges of continuous predictors, like trial number. Thus I use estimated value at the trial that is one-sixth into the post-test to estimate classification functions, rather than the very beginning, because it is close to the beginning of the post test, but not so close that the predictions are substantially affected by the extra instability of the predictions that come at the edge of a continuous predictors range.

${ }^{13}$ As above, these are Bayesian posterior $p$ values, or the proportion of posterior samples in which the inferred boundary was
} 


\section{Experiment 4: Category boundaries}

Estimated values at first sixth of test block

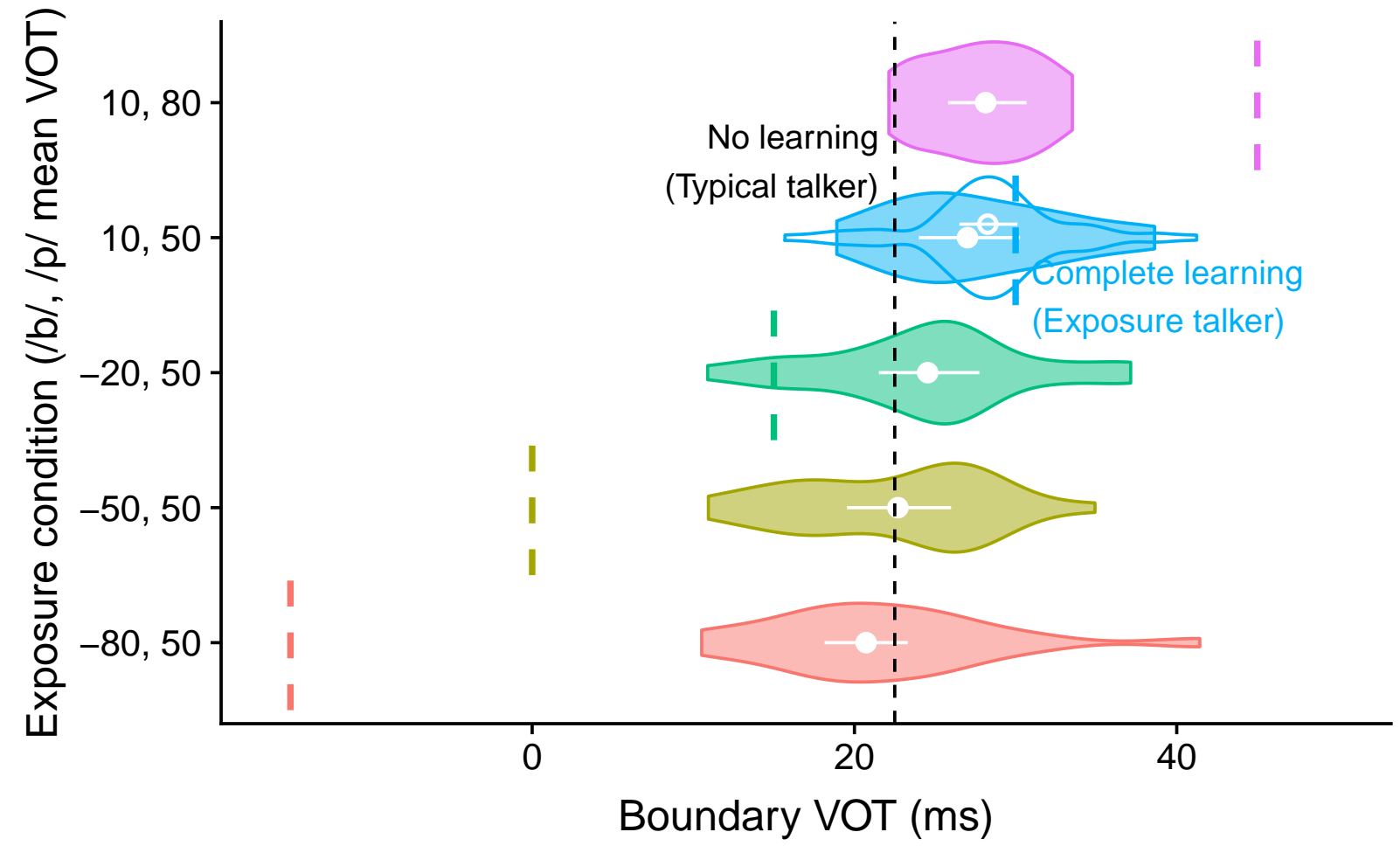

Figure 15: /b/-/p/ category boundaries from Experiment 4. Violins show distributions of subject's boundaries (estimated based on Bayesian mixed effect logistic regression) and points/CIs show the posterior mean and $95 \%$ CI for the group-level (fixed effect) estimate from the same regression. Subjects' boundaries from the Experiment 1 condition with the same exposure statistics are shown in outline, and are quite close to the results from Experiment 4 (if less variable). Note that the position of the 'complete learning' boundaries (thick dashed lines; based on the exposure statistics) are much more extreme than in Experiment 1, reflecting the more extreme shifts in the distributions that listeners experience in Experiment 4.

$-50 \mathrm{~ms}$ vs. $-80 \mathrm{~ms} / \mathrm{b} /$ ). Even comparing the $-80 \mathrm{~ms} / \mathrm{b} /$ and $-20 \mathrm{~ms} / \mathrm{b} /$ conditions, the difference between the boundaries is more reliable but still small in absolute terms and with a credible interval which contains 0 , only $3.8 \mathrm{~ms}$ (95\% credible interval $[-0.25,8])$.

However, this lack of robust distributional learning in this experiment is not unique to large negative VOTs. The $10 \mathrm{~ms} / \mathrm{b} /, 80 \mathrm{~ms} / \mathrm{p} /$ mean condition also produces comparably small shifts in category boundaries compared with the $10 \mathrm{~ms} / \mathrm{b} /, 50 \mathrm{~ms} / \mathrm{p} /$ condition, with a posterior mean difference between the populationlevel boundaries of only $1.1 \mathrm{~ms}$ (95\% credible interval $[-2.7,4.8])$. Compare this with the $15 \mathrm{~ms}$ difference between the boundaries implied by the exposure distributions (30ms vs. $45 \mathrm{~ms}$ VOT).

Finally, can the simple belief updating model from Experiment 3-using the prior beliefs inferred from Experiment 1 - explain the distributional learning or lack thereof observed in this experiment? Figure 16 shows the posterior predictive intervals for the model compared with the average probability of $/ \mathrm{p} /$ responses in the first third of the test phase of Experiment 4. The model's predictions are quite accurate for the 10ms $/ \mathrm{b} /, 50 \mathrm{~ms} / \mathrm{p} /$ condition which is a near replication of the same exposure condition from Experiment 1. But for the other conditions, the model tends to predict more distributional learning (larger positive or negative boundary shifts) than listeners actually show, or in the case of the two most extreme negative VOT

higher for the condition with the higher exposure mean. 


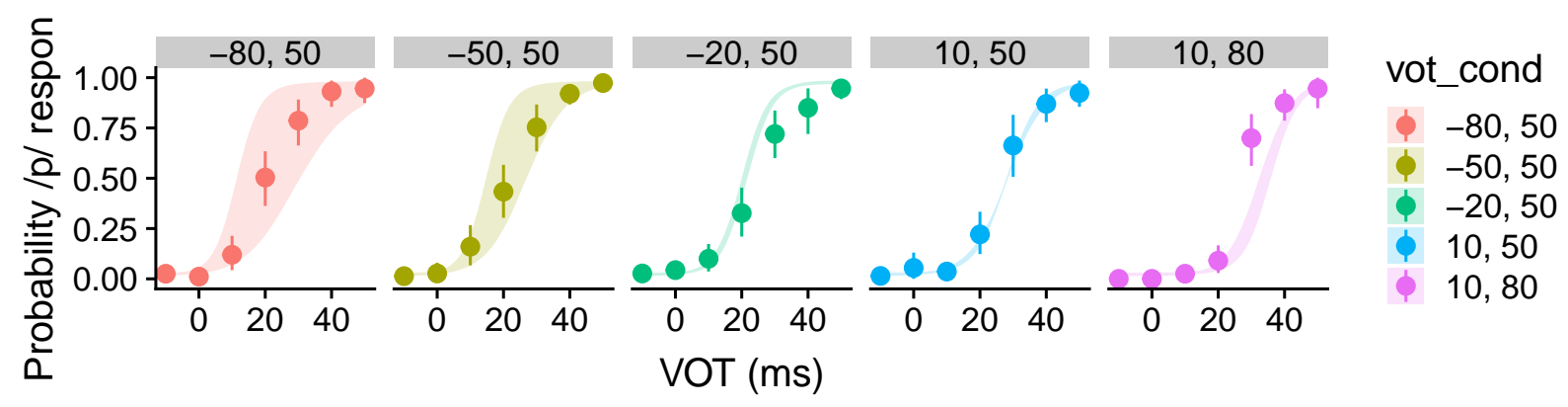

Figure 16: The actual categorization functions observed in this experiment (dots and lines) diverge from the predictions of the simple belief updating model from Experiment 3 (shaded regions), which are based on prior beliefs inferred based on distributional learning in Experiment 1. Shaded regions show the 95\% posterior predictive intervals for the belief updating model, based on prior beliefs inferred from Experiment 1 and exposure distributions from Experiment 2. Dots and errorbars show mean and $95 \%$ bootstrapped CIs for the mean probability of $/ \mathrm{p} /$ response over subjects. Note the additional uncertainty in predictions relative to the posterior predictive distribution given Experiment 1's conditions (Figure 8).

conditions, makes very vague predictions.

\subsection{Discussion}

On the one hand, the results from Experiment 4 are qualitatively consistent with the earlier experiments: distributional learning is constrained, and the limited learning (change in category boundaries) observed here is consistent with the large differences between the exposure distributions and a typical talker's VOT distributions. On the other hand, in Experiments 1 and 2 there was some distributional learning even for the most shifted distributions, whereas in this experiment exposure to increasingly negative VOTs led to minimal (and statistically unreliable) changes in listeners' category boundaries. Likewise, there was also little difference in category boundaries after exposure to a $/ \mathrm{p} /$ mean of $50 \mathrm{~ms}$ vs. $80 \mathrm{~ms}$ VOT (with /b/ mean of $10 \mathrm{~ms}$ VOT). Moreover, the quantitative predictions of a Bayesian belief updating model — based on the pattern of learning observed in Experiment 1 - do not match the limited learning observed here.

There are a number of possible reasons for these discrepancies. Here, I discuss two possible explanations: first, differences in the VOT distributions listeners were exposed to, and second, methodological differences in how boundaries were assessed.

\subsubsection{Differences in exposure distributions}

In this experiment, listeners were exposed to VOT distributions that differed in important ways from Experiments 1 and 2. First, they deviated even more dramatically from the distributions produced by a typical talker of American English. In particular, some conditions included large negative VOTs, for which little distributional learning was observed. The lack of reliable distributional learning from negative VOTs is consistent with Sumner (2011), who also found that negative VOTs failed to produce large shifts in category boundaries except in a few particular presentation orders. Moreover, as I discuss below, the belief updating model makes a basic assumption that there are exactly two clusters of VOT for word-initial stops, which may prevent it from effectively predicting the (lack of) distributional learning of negative VOTs.

Second, in Experiments 1 and 2 the means of the high and low VOT clusters moved together in lockstep, while in this experiment they were manipulated independently. Varying the means of each cluster together maximizes the necessity of a boundary shift, since failure to shift the boundary would result in a large increase in the rate of errors given the implied category assignments from the clusters. Exposure distributions where only one cluster deviates from listeners expectations do not require as large of a shift in the boundary between $/ \mathrm{b} /$ and $/ \mathrm{p} /$ in order to achieve a low recognition error. In fact, for all the exposure distributions tested in Experiment 4, continuing to use the ideal boundary for a "typical talker" would yield 
low error rates (less than $1.4 \%$ in all conditions; see Figure 13). Thus the potential benefit of distributional learning was lower in this experiment than in Experiments 1 and 2. If listeners are doing boundedly rational belief updating under some kind of resource constraints, distributional learning might only lead to changes in behavior when the cost of changing behavior is outweighed by the benefit of any such change (Tavoni, Balasubramanian, \& Gold, 2019)

\subsubsection{Methodological differences}

There are also important differences in the procedure used to assess distributional learning which could explain the overall weaker distributional learning in this experiment. Experiments 1 and 2 did not distinguish between an exposure and test phase, and measured distributional learning via responses to trials during the bimodally distributed exposure. In Experiment 4, the VOT clusters in the exposure distributions were so widely separated that in many conditions no trials had VOTs near the expected boundary, and so a separate post-test phase was necessary to measure listeners' categorization functions. In this phase, listeners experienced a very different distribution of VOTs which was flat between -10ms and 50ms. Thus, it's possible that distributional learning continued throughout the test phase, undoing some of what listeners had learned from the earlier bimodal distributions (and indeed there is some evidence that the small differences between conditions present at the beginning of the test phase gradually disappear, see Supplementary Materials).

More generally, the bimodal exposure distributions in Experiment 4 provide no direct evidence about how the range of VOTs during test $(-10 \mathrm{~ms}$ to $50 \mathrm{~ms}$ VOT) should be categorized. The one exception to this is the $10 \mathrm{~ms} / \mathrm{b} /, 50 \mathrm{~ms} / \mathrm{p} /$ condition, which is the only condition that showed a comparable degree of distributional learning as the earlier experiments. The evidence provided by the exposure distributions in any of these experiments may not seem to be direct in the sense that there was no explicit labeling of any of the VOTs as $/ \mathrm{b} /$ or $/ \mathrm{p} /$. Nevertheless, tokens that were encountered during test actually occurred during exposure in Experiments 1 and 2, whereas in this experiment listeners are hearing many of the VOTs of the test phase for the first time during the test phase itself. Thus, they have to extrapolate from their experience with other, quite different VOTs, and this extrapolation is much more sensitive to particular underlying assumptions of the speech perception system (or my model thereof) than the interpolation of Experiments 1 and 2.

\section{General Discussion}

This paper has explored the nature and possible explanations of constraints on distributional learning in adults. Experiment 1 shows that distributional learning of voice onset time (VOT) as a cue to word-initial stop voicing is constrained: listeners learn some distributions ("accents") better than others, as measured by the agreement between their actual $/ \mathrm{b} /-/ \mathrm{p} /$ category boundary and the boundary that is implied by the distributions they are exposed to. Moreover, listeners show more complete learning for distributions that are closer to what a typical talker of American English produces, which suggests that listeners are drawing on their previous experience with other talkers to guide their learning. Experiment 2 tests an alternative hypothesis: that these constraints are really due to the fact that the typical distributional learning task is unsupervised, and that the apparent constraints found in Experiment 1 would go away when some VOTs are labeled according to the intended category. Surprisingly, adding labeling information to half the trials had no effect on distributional learning. Experiment 3 shows that a belief updating model - where all listeners start from the same prior beliefs - can capture the pattern of distributional learning (or lack thereof) found in Experiment 1. Moreover, the model's inferred prior beliefs are similar to the VOT distributions that a typical talker of English produces. This suggests that the constraints on distributional learning observed in Experiment 1 are at least consistent with belief updating guided by prior experience with other talkers. In the remainder of this paper, I will discuss the implications of these results for future work, and the caveats given a final Experiment 4, which suggests that the constraints on distributional learning of VOT/voicing may be more complex than revealed in the first three experiments.

\subsection{Is distributional learning (Bayesian) belief updating?}

The evidence from Experiments 1-3 provides clear support for the idea that constraints on distributional learning arise from prior experience with talker variability. Nearly all listeners use category boundaries that 
are a compromise between the optimal boundaries for the VOT distributions of a typical talker and those of the exposure talker, and a Bayesian belief-updating model can explain the particular pattern of distributional learning or lack thereof given prior beliefs that are similar to the VOT distributions produced by a typical talker of American English.

However, this same model, given prior beliefs that explain distributional learning in Experiment 1, fails to accurately predict distributional learning to different VOT distributions in Experiment 4. Does this mean that the belief-updating hypothesis is falsified? In evaluating any computational model, it is important to keep in mind that successful prediction or generalization depends not only on suitability of the theory that the model instantiates, but also on the suitability or approximate correctness of every one of a long chain of linking hypotheses, computational simplifications, and technical assumptions that connect the theory to behavior. If only a single link in this chain is broken, then the model may not generalize. In the discussion of Experiment 4 above I discussed some methodological differences between the two experiments that might explain the observed failure to generalize. Here, I will discuss some relevant specific assumptions of the model itself.

\subsubsection{Potential points of failure in the belief updating model}

One of the basic assumptions of the belief updating model tested here is that there are exactly two clusters of VOTs for word initial stops: one corresponding to the voiced stop /b/ and a second for voiceless /p/. However, even though American English has two phonemic voicing categories, many talkers produce three distinct clusters of VOTs, with a single long-lag VOT cluster for voiceless (aspirated) stops, and two clusters for phonemically voiced stops: one with a mean around 0ms VOT and low variance (short-lag), and another with a large negative mean VOT and high variance (pre-voiced) (e.g. Goldrick et al., 2013; Lisker \& Abramson, 1964). In an ideal listener model with these three VOT clusters, the short-lag cluster would largely determine the voiced/voiceless category boundary, since the pre-voiced and short-lag clusters are both phonemically voiced. When presented with un-labeled VOTs, a fully ideal adapter model would simultaneously classify VOTs and update beliefs about the underlying distributions, weighted by how likely each distribution is to explain that observation (see e.g. Kleinschmidt \& Jaeger, 2015, eq. 7, p. 155). Starting from prior beliefs based on a typical talker of American English, such a model would likely assign large negative VOTs to the pre-voiced cluster, leading to minimal change in beliefs about the un-aspirated cluster and hence minimal changes in categorization.

While this is a conceptually straightforward prediction of the theory that distributional learning is a form of Bayesian belief-updating, testing this in an implemented model presents a number of technical challenges. Not least among them is the fact that the model presented in Experiment 3 is not a fully ideal adapter which classifies and adapts tokens at the same time, but rather assumes that the cluster labels of each token are known. This assumption massively simplifies the inference in the model, and is reasonable given not only that the bimodal structure of the exposure distributions is extremely clear, but also that Experiment 2 found that providing cluster labels to many tokens led to no change in distributional learning. But it's unclear what the cluster labels should be when there are three clusters (two for phonemically voiced stops), so I leave exploring this possibility for future work. One promising direction would be to use sequential Monte Carlo algorithms to approximate the online inference required to simultaneously classify and adapt (see e.g. Kleinschmidt, 2018; Sanborn, Griffiths, \& Navarro, 2010).

The other major simplifying assumption of the belief updating model which may be suspect is the form of the prior beliefs themselves. Specifically, the model assumes that the prior beliefs about $/ \mathrm{b} / \mathrm{and} / \mathrm{p} /$ are independent, and that each distribution has a conjugate prior. Chodroff and Wilson (2017) found strong correlations between the mean VOTs for voiceless stops with different places of articulation across talkers, and weaker but significant correlations between the means for voiced and voiceless stops (at least for spontaneous speech). Independent priors for different voiced stops cannot capture the actual structure of variation across talkers, and thus may not be a good approximation of listeners' prior beliefs. Furthermore, the use of a conjugate prior (again, for computational reasons) and results in a restricted set of possible relationships between expectations about within- and between-talker variance in VOT. 


\subsubsection{Long-term trajectory of distributional learning}

Finally, a more serious problem for the belief updating model used here is the long-term distributional learning behavior. Specifically, a straightforward Bayesian belief-updating model predicts that no matter how strong the prior beliefs, they can eventually be overcome by observed data. This is a consequence of treating every observation as equally informative about the current distributions. The empirical evidence on this question is mixed, with some studies finding that listeners continue to integrate evidence even over rather dramatic changes in context (e.g. Kraljic \& Samuel, 2006, 2007; Theodore \& Monto, 2019), while others find that listeners seem to weight more recent evidence more strongly, even within the context of a single talker (Olasagasti \& Giraud, 2018; Saltzman \& Myers, 2017).

These findings may not be compatible with the simple model presented here, but they are compatible with the theory of Bayesian belief-updating. They simply correspond to belief updating models which make different assumptions about the generative process. If the generative process is such that the distribution parameters (mean, variance, etc.) vary across contexts (e.g., talkers) but are stable within talkers, then each token that a talker produces should be treated as equally informative. However, if the generative process is instead one where there is some amount of drift, variability, or change over time even within a context (talker), then a Bayesian belief updating model would weight more recent observations more heavily, since according to the assumed generative process they come from distributions with parameters that are more similar to the current ones.

The empirical data from these studies is not clear enough about the learning curves over time to be able to resolve this question unambiguously. However, visually inspecting the learning curves predicted by the belief updating model compared with the (admittedly crude) measurements of the actual learning curves (Figure 9) suggests that learning may be initially more rapid and asymptote more quickly than this particular belief updating model captures. If this is in fact the case, then in a belief-updating framework it would suggest that listeners assume there can be some local variation within a talker, in addition to global variation across talkers. More work that examines the time course of adaptation more carefully is needed to better understand these dynamics and further constrain models of adaptation. A final but important caveat for this work is that the time course and assumed generative model may be different for different parts of the linguistic generative model. The "ideal adapter" framework of Kleinschmidt and Jaeger (2015), as well as, Qian, Jaeger, and Aslin (2012, July) points out that the kind of adaptation a system does is itself something that should be adapted to the amount and kind of variability that's present in the environment, and if different aspects of the linguistic generative model have different amounts and kinds of variability, we should expect adaptation to have different characteristics as well.

\subsection{Implications for future work}

The first, most general implication of these results is that they suggest a view where distributional learning operates continuously throughout language acquisition and adult language use and adaptation. The idea that statistical learning continues throughout the lifespan is not a new idea (e.g., Botvinick \& Plaut, 2004; Chang, Dell, \& Bock, 2006; Elman, 1990; Goldinger, 1998; K. Johnson, 1997; Nielsen \& Wilson, 2008), although it has received more attention of late in the study of language processing (Fine \& Jaeger, 2013; Kleinschmidt \& Jaeger, 2015; Schuster \& Degen, 2020; Yildirim, Degen, Tanenhaus, \& Jaeger, 2016). Nor is it specific to language (e.g., Qian et al., 2012, July): at a basic level, the sensitivities of perceptual systems are tuned to both the long-run statistical properties of the environment (e.g., Ganguli \& Simoncelli, 2016; Simoncelli \& Olshausen, 2001) and short-run changes in those properties (e.g., Schweinhart, Shafto, \& Essock, 2017; Stocker \& Simoncelli, 2006).

In this view, acquisition and adaptation are both forms of distributional learning, with the primary difference between them being the different amounts and types of prior experience that the system can draw on. The role of prior experience in constraining distributional learning hints at another sense in which acquisition and adaptation may be connected by distributional learning. In order for prior experience with talker variability to constrain adaptation in a belief updating model, the listener must have learned something about the patterns of variability across talkers. At a computational level (in the sense of Marr, 1982), this may also be a form of distributional learning. In order to effectively and efficiently adapt to an unfamiliar talker's accent, a listener needs to have some reasonably good estimate of the amount and kind of talker 
variability they should expect, which is directly related to the distribution of talkers' accents $^{14}$ that exist in their environment. In this view - articulated in Kleinschmidt and Jaeger (2015) - not only are adaptation and acquisition both forms of distributional learning, but the difference between them is due to continuous distributional learning at another level. The experiments reported here are at least consistent with this view: distributions that diverge more from a typical talker's distributions are harder to learn. The other existing evidence for this claim was reviewed in Kleinschmidt and Jaeger (2015), and I will not revisit it here in depth. However, one additional piece of evidence for this possibility is that learning at the second level (of talker variability) appears to operate continuously during language acquisition, and in parallel with acquisition of the linguistic structure (for a recent review, see E. K. Johnson \& White, 2020).

This possibility raises two related sets of questions for future research: (1) what distributions of talkers' accents are available for learning, and (2) do listeners' distributional learning biases and constraints reflect such meta-distributions (distributions of accents across talkers) that they have experienced?

\subsubsection{What distributions are available to learn?}

The first question can be answered by looking at the amount and structure of the talker variability that actually exists in the world. Current attempts at this have already revealed interesting findings. For instance, vowel formant distributions vary little within a talker (over the course of a day), but vary considerably across talkers (Heald \& Nusbaum, 2015). Vowels are more variable across talkers than voicing of stop consonants, even once variability in vocal tract length is taken into account (Kleinschmidt, 2019). Moreover, this variability is in many cases highly structured within a talker, across categories (Chodroff \& Wilson, 2017).

When combined with a Bayesian belief updating model like the one employed here, these findings suggest clear predictions for constraints on distributional learning during adaptation. If talkers are consistent in their realization of vowels, learning should be relatively stable and accumulate evidence over time. If vowels are more variable than stop voicing, listeners should show less constrained, more strongly experience-driven distributional learning of vowels than stop voicing. If certain phonemes' distributions are correlated within talkers, listeners should have a harder time learning distributions that violate these correlations (e.g., a higher-than average mean VOT for /p/ but lower-than-average mean VOT for /k/).

Thus far, the studies of variability in production are only scratching the surface of the possible structures that might exist in talker variability. Investigating differences across languages and dialects is particularly pressing. To what extent are these patterns universal across languages, rather than language- or dialectspecific (e.g., Schertz \& Kang, 2016)? Language-specific patterns in talker variability must, by definition, be learned during language acquisition, greatly adding to the burden on the learner.

\subsubsection{What do people know about talker variability?}

One of the major challenges in this research program is to probe the implicit, subjective expectations that listeners bring when adapting to an unfamiliar talker. These beliefs are difficult to probe because we can only ever observe with any amount of directness a listener's beliefs about the current context. These beliefs combine their implicit expectations with their recent experience, and so any of the measurements we can make in an experiment provide only indirect evidence about those prior expectations. Some kind of linking hypothesis is needed in order to be able to assess these implicit prior expectations.

One possible such linking hypothesis is the kind of belief updating model presented in Experiment 3. This sort of model, along with data on the degree and specific type of distributional learning in different exposure conditions, could in principle serve as a kind of "mind reading" device, allowing us to probe listeners' subjective prior beliefs. This is a straightforward application of the principles of Bayesian cognitive modeling: there is some unobservable cognitive variables (subjective prior beliefs), and Bayesian inference allows us to infer them based on behavior. This procedure did yield some meaningful information (beyond the simple "existence proof" of the suitability of belief updating as a model for distributional learning in this experiment). First, the model tells us something about how confident listeners are in their prior beliefs. Second, the model tells us something about the content of those beliefs, the specific expectations about

\footnotetext{
${ }^{14}$ Using "accent" here as a shorthand for the probabilistic generative model that characterizes an individuals use of their language.
} 
the mean and variance of the VOT distributions for $/ \mathrm{b} /$ and $/ \mathrm{p} /$ that are most consistent with the overall pattern of distributional learning.

However, in practice, these inferences are rather more tenuous than we might like, since as discussed above they depend on all of the various assumptions that the model makes in linking behavior to the cognitive variables of interest. Some assumptions are fundamental to the logical structure of the model, but some are simplifications made for reasons of mathematical or computational tractability. For instance, one important assumption was that no information about the actual underlying distributions of VOTs for $/ \mathrm{b} /$ and $/ \mathrm{p} /$ (or their variability across talkers) was built in. Doing so would be straightforward (as a "hyperprior" in the belief updating model), but would also mean that the inferred prior beliefs would be colored by our own assumptions as scientists about what VOT distributions should look like, negating somewhat the usefulness of this technique for "mind reading".

The tenuousness of the inferences about listeners prior beliefs is demonstrated by the model's poor performance when predicting behavior in Experiment 4. This was a stringent, but critically important test. If the model really had, in fact, recovered listener's prior expectations about what kind of VOT distributions an unfamiliar talker is likely to produce, then it should be able to predict distributional learning even to distributions it was not trained on. This was clearly not the case in Experiment 4, which means that one or more of the many linking hypotheses connecting this model to the experimental data is incorrect. While this calls for some caution in using such a Bayesian belief-updating model as a mind-reading tool or even as an explanation of distributional learning, it also provides a number of potential directions for future work, both computationally (e.g., investigating the consequences of the various simplifications made here) and empirically (e.g., investigating learning from even other distributions, with multiple cues, and under varying training regimes; see Harmon, Idemaru, \& Kapatsinski, 2019, for work in this direction).

\subsubsection{What is the role of specific language experience?}

Finally, the evidence I have presented here is circumstantial: I observe constraints on distributional learning of VOT among American English listeners, and I have shown that these constraints are largely consistent with belief-updating starting from prior beliefs that look very similar to a typical talker of American English. Moreover, the VOT distributions that the American English listeners struggled to learn - while atypical of American English - are not outside the range of VOT distributions found across languages (Cho et al., 2019; Lisker \& Abramson, 1964). However, more work is necessary to show directly that it is previous experience with other talkers that constrains distributional learning. One step in this direction would be comparing learning of the same distributions by listeners with differing language experiences, provided that that experience differs enough in the relevant cue distributions. Another important step would be showing that specific experience or specific prior expectations can further constrain distributional learning, although this may be difficult since the strong constraints exhibited in these experiments likely reflect language-level priors which cannot be easily or quickly changed (Kleinschmidt \& Jaeger, 2015)

\section{Conclusion}

One of the major discoveries of the last two decades of research in speech processing is that adults maintain a surprising amount of flexibility, and quickly adapt to unusual variants of sounds in their language. Moreover, this rapid adaptation may correspond to the continued operation of the same kind of distributional learning mechanisms which have been implicated in language acquisition early in life (Kleinschmidt \& Jaeger, 2015). In this paper, I have explored some constraints on the ability of distributional learning in adults, finding that adult distributional learning is constrained, these constraints do not appear to arise from the unsupervised nature of distributional learning, but rather are consistent with belief updating guided by a listener's prior experience with talker variability.

\section{Acknowledgments}

This work was begun during my PhD studies at the University of Rochester and an early version formed one chapter of my dissertation. In addition, some of the data reported here was presented at the Annual 
Meeting of the Cognitive Science Society (2015 and 2016). I gratefully acknowledge my PhD advisor (Florian Jaeger), my committee (Dick Aslin, Jeff Runner, and Lori Holt), and Rajeev Raizada for their suggestions and careful critiques in the early phases of developing this work.

I am also indebted to the developers and maintainers of the R language (R Core Team, 2020), the Stan language (Carpenter et al., 2017), and the following R packages: brms (Bürkner, 2017), knitr (Xie, 2018), rmarkdown (Allaire et al., 2018), tidyverse (Wickham, 2017), dplyr (Wickham, François, Henry, \& Müller, 2018), glue (Hester, 2018), magrittr (Bache \& Wickham, 2014), forcats (Wickham, 2018), rstan (Guo, Gabry, \& Goodrich, 2018), loo (Vehtari, Gabry, Magnusson, Yao, \& Gelman, 2019), tidybayes (Kay, 2018), ggplot2 (Wickham, Chang, et al., 2018), cowplot (Wilke, 2018), and latex2exp (Meschiari, 2015).

This work was supported by an NSF GRFP and NIH NICHD F31 (HD082893) to DFK and NIH NICHD R01 (HD075797) to TFJ.

\section{References}

Allaire, J., Xie, Y., McPherson, J., Luraschi, J., Ushey, K., Atkins, A., ... Chang, W. (2018). Rmarkdown: Dynamic documents for $r$. R package version 1.10. Retrieved from https://CRAN.R-project.org/ package $=$ rmarkdown

Allen, J. S., Miller, J. L., \& DeSteno, D. (2003). Individual talker differences in voice-onset-time. The Journal of the Acoustical Society of America, 113(1), 544-544. doi:10.1121/1.1528172

Bache, S. M., \& Wickham, H. (2014). Magrittr: A forward-pipe operator for $r$. R package version 1.5. Retrieved from https://CRAN.R-project.org/package=magrittr

Baese-berk, M. M., Bradlow, A. R., \& Wright, B. A. (2013). Accent-independent adaptation to foreign accented speech. JASA Express Letters, 133(3), 174-180. doi:10.1121/1.4789864

Bejjanki, V. R., Clayards, M. A., Knill, D. C., \& Aslin, R. N. (2011). Cue integration in categorical tasks: Insights from audio-visual speech perception. PLoS ONE, 6(5), e19812-e19812. doi:10.1371/journal. pone.0019812

Bergelson, E., \& Swingley, D. (2012). At 6-9 months, human infants know the meanings of many common nouns. Proceedings of the National Academy of Sciences, 109(9), 3253-3258. doi:10.1073/pnas. 1113380109. pmid: 22331874

Bertelson, P., Vroomen, J., \& de Gelder, B. (2003). Visual recalibration of auditory speech identification: A McGurk aftereffect. Psychological Science, 14(6), 592-597. doi:10.1046/j.0956-7976.2003.psci_1470.x

Best, C. T. [Catherine T.], McRoberts, G. W., LaFleur, R., \& Silver-Isenstadt, J. (1995). Divergent developmental patterns for infants' perception of two nonnative consonant contrasts. Infant Behavior and Development, 18(3), 339-350. doi:10.1016/0163-6383(95)90022-5

Best, C. T. [Catherine T], McRoberts, G. W., Gerald W., \& Sithole, N. M. (1988). Examination of perceptual reorganization for nonnative speech contrasts: Zulu click discrimination by English-speaking adults and infants. Journal of Experimental Psychology. Human Perception and Performance, 14(3), 345-360. pmid: 2971765

Botvinick, M. M., \& Plaut, D. C. (2004). Doing without schema hierarchies: A recurrent connectionist approach to normal and impaired routine sequential action. Psychological Review, 111(2), 395-429. doi:10.1037/0033-295X.111.2.395. PMID: 15065915

Bradlow, A. R., \& Bent, T. (2008). Perceptual adaptation to non-native speech. Cognition, 106(2), 707-29. doi:10.1016/j.cognition.2007.04.005

Bradlow, A. R., Pisoni, D. B., Akahane-Yamada, R., \& Tohkura, Y. (1997). Training Japanese listeners to identify English /r/ and /l/: IV. Some effects of perceptual learning on speech production. The Journal of the Acoustical Society of America, 101(4), 2299-310. PMID: 9104031

Bürkner, P.-C. (2017). Brms: An R Package for Bayesian Multilevel Models Using Stan. Journal of Statistical Software, 80(1), 1-28. doi:10.18637/jss.v080.i01

Carpenter, B., Gelman, A., Hoffman, M. D., Lee, D., Goodrich, B., Betancourt, M., ... Riddell, A. (2017). Stan: A Probabilistic Programming Language. Journal of Statistical Software, 76(1). doi:10.18637/ jss.v076.i01

Chang, F., Dell, G. S., \& Bock, K. (2006). Becoming syntactic. Psychological Review, 113(2), 234-72. doi:10. 1037/0033-295X.113.2.234. PMID: 16637761 
Chládková, K., Podlipský, V. J., \& Chionidou, A. (2017). Perceptual adaptation of vowels generalizes across the phonology and does not require local context. Journal of Experimental Psychology: Human Perception and Performance, 43(2), 414-427. doi:10.1037/xhp0000333

Cho, T., Whalen, D. H., \& Docherty, G. (2019). Voice onset time and beyond: Exploring laryngeal contrast in 19 languages. Journal of Phonetics, 72, 52-65. doi:10.1016/j.wocn.2018.11.002

Chodroff, E., \& Wilson, C. (2017). Structure in talker-specific phonetic realization: Covariation of stop consonant VOT in American English. Journal of Phonetics, 61, 30-47. doi:10.1016/j.wocn.2017.01.001

Clarke-Davidson, C. M., \& Garrett, M. F. (2004). Rapid adaptation to foreign-accented English. The Journal of the Acoustical Society of America, 116(6), 3647-3647. doi:10.1121/1.1815131

Clayards, M. A., Tanenhaus, M. K., Aslin, R. N., \& a Jacobs, R. (2008). Perception of speech reflects optimal use of probabilistic speech cues. Cognition, 108(3), 804-9. doi:10.1016/j.cognition.2008.04.004

Clopper, C. G., Pisoni, D. B., \& de Jong, K. J. (2005). Acoustic characteristics of the vowel systems of six regional varieties of American English. The Journal of the Acoustical Society of America, 118(3), 1661-1676. doi:10.1121/1.2000774

Colby, S., Clayards, M., \& Baum, S. (2018). The Role of Lexical Status and Individual Differences for Perceptual Learning in Younger and Older Adults. Journal of Speech Language and Hearing Research, 61(8), 1855. doi:10.1044/2018_JSLHR-S-17-0392

Cristià, A. (2018). Can infants learn phonology in the lab? A meta-analytic answer. Cognition, 170, 312-327. doi:10.1016/j.cognition.2017.09.016

Elman, J. L. (1990). Finding Structure in Time. Cognitive Science, 14(2), 179-211. doi:10.1207/s15516709cog1402_ 1

Ernst, M. O., \& Bülthoff, H. H. (2004). Merging the senses into a robust percept. Trends in Cognitive Sciences, 8(4), 162-9. doi:10.1016/j.tics.2004.02.002. PMID: 15050512

Escudero, P., Benders, T., \& Wanrooij, K. (2011). Enhanced bimodal distributions facilitate the learning of second language vowels. The Journal of the Acoustical Society of America, 130(4), EL206-EL212. doi:10.1121/1.3629144

Feldman, N. H., Griffiths, T. L., Goldwater, S., \& Morgan, J. L. (2013). A role for the developing lexicon in phonetic category acquisition. Psychological Review, 120(4), 751-778. doi:10.1037/a0034245

Feldman, N. H., Myers, E. B., White, K. S., Griffiths, T. L., \& Morgan, J. L. (2013). Word-level information influences phonetic learning in adults and infants. Cognition, 127(3), 427-438. Retrieved from http: //ling.umd.edu/ nhf/papers/LexicalDistributionalLong.pdf

Fine, A. B., \& Jaeger, T. F. (2013). Evidence for Implicit Learning in Syntactic Comprehension. Cognitive Science, 37(3), 578-591. doi:10.1111/cogs.12022

Ganguli, D., \& Simoncelli, E. P. (2016). Neural and perceptual signatures of efficient sensory coding, 1-24. Retrieved from http://arxiv.org/abs/1603.00058

Gelman, A., \& Hill, J. (2007). Data analysis using regression and multilevel/hierarchical models. Cambridge University Press New York.

Goldinger, S. D. (1998). Echoes of echoes? An episodic theory of lexical access. Psychological Review, 105(2), 251-79. PMID: 9577239

Goldrick, M., Vaughn, C., \& Murphy, A. (2013). The effects of lexical neighbors on stop consonant articulation. The Journal of the Acoustical Society of America, 134(2), EL172-7. doi:10.1121/1.4812821. PMID: 23927221

Goto, H. (1971). Auditory perception by normal Japanese adults of the sounds "L" and "R". Neuropsychologia, 9(3), 317-323. doi:10.1016/0028-3932(71)90027-3

Goudbeek, M., Cutler, A., \& Smits, R. (2008). Supervised and unsupervised learning of multidimensionally varying non-native speech categories. Speech Communication, 50(2), 109-125. doi:10.1016/j.specom. 2007.07.003

Guo, J., Gabry, J., \& Goodrich, B. (2018). Rstan: R interface to stan. R package version 2.17.3. Retrieved from https://CRAN.R-project.org/package $=$ rstan

Harmon, Z., Idemaru, K., \& Kapatsinski, V. (2019). Learning mechanisms in cue reweighting. Cognition, 189, 76-88. doi:10.1016/j.cognition.2019.03.011

Hartshorne, J. K., Tenenbaum, J. B., \& Pinker, S. (2018). A critical period for second language acquisition: Evidence from 2/3 million English speakers. Cognition, 177, 263-277. doi:10.1016/j.cognition.2018.04. 007 
Heald, S. L. M., \& Nusbaum, H. C. (2015). Variability in vowel production within and between days. PLoS ONE, 10(9), e0136791-e0136791. doi:10.1371/journal.pone.0136791

Hester, J. (2018). Glue: Interpreted string literals. R package version 1.3.0. Retrieved from https://CRAN.Rproject.org $/$ package $=$ glue

Hitczenko, K., Mazuka, R., Elsner, M., \& Feldman, N. H. (2018). How to use context to disambiguate overlapping categories: The test case of Japanese vowel length. In C. Kalish, M. Rau, J. Zhu, \& T. T. Rogers (Eds.), Proceedings of the 40th Annual Meeting of the Cognitive Science Society, Austin, TX: Cognitive Science Society.

House, A. S. (1953). The Influence of Consonant Environment upon the Secondary Acoustical Characteristics of Vowels. The Journal of the Acoustical Society of America, 25(1), 105-105. doi:10.1121/1.1906982

Idemaru, K., \& Holt, L. L. (2011). Word recognition reflects dimension-based statistical learning. Journal of Experimental Psychology: Human Perception and Performance, 37(6), 1939-56. doi:10.1037/a0025641

Johnson, E. K., \& White, K. S. (2020). Developmental sociolinguistics: Children's acquisition of language variation. WIREs Cognitive Science, 11(1). doi:10.1002/wcs.1515

Johnson, K. (1997). Speech perception without speaker normalization: An exemplar model. In Johnson, K \& Mullennix, J W (Eds.), Talker Variability in Speech Processing (pp. 145-165). San Diego: Academic Press.

Kass, R. E., \& Raftery, A. E. (1995). Bayes Factors. Journal of the American Statistical Association, 90(430), 773-795. doi:10.1080/01621459.1995.10476572

Kay, M. (2018). Tidybayes: Tidy data and 'geoms' for bayesian models. R package version 1.0.1. Retrieved from https://CRAN.R-project.org/package=tidybayes

Keetels, M., Schakel, L., Bonte, M., \& Vroomen, J. (2016). Phonetic recalibration of speech by text. Attention, Perception, \& Psychophysics, 78(3), 938-945. doi:10.3758/s13414-015-1034-y

Klatt, D. H. (1980). Software for a cascade/paralell formant synthesizer. Journal of the Acoustical Society of America, 67(3), 971-995. doi:10.3758/BRM.42.3.863

Kleinschmidt, D. F. (2018). Learning distributions as they come: Particle filter models for online distributional learning of phonetic categories. In T. T. Rogers, M. Rau, J. Zhu, \& C. Kalish (Eds.), Proceedings of the 40th Annual Conference of the Cognitive Science Society (pp. 1933-1938). doi:10.31234/osf.io/ dymc8

Kleinschmidt, D. F. (2019). Structure in talker variability: How much is there and how much can it help? Language, Cognition and Neuroscience, 34(1), 43-68. doi:10.1080/23273798.2018.1500698

Kleinschmidt, D. F., \& Jaeger, T. F. (2015). Robust speech perception: Recognize the familiar, generalize to the similar, and adapt to the novel. Psychological Review, 122(2), 148-203. doi:10.1037/a0038695

Kohler, K. J. (1982). F0 in the production of lenis and fortis plosives. Phonetica, 39(4-5), 199-218. doi:10. $1159 / 000261663$

Kraljic, T., \& Samuel, A. G. (2005). Perceptual learning for speech: Is there a return to normal? Cognitive Psychology, 51(2), 141-78. doi:10.1016/j.cogpsych.2005.05.001. PMID: 16095588

Kraljic, T., \& Samuel, A. G. (2006). Generalization in perceptual learning for speech. Psychonomic Bulletin G Review, 13(2), 262-8. PMID: 16892992

Kraljic, T., \& Samuel, A. G. (2007). Perceptual adjustments to multiple speakers. Journal of Memory and Language, 56(1), 1-15. doi:10.1016/j.jml.2006.07.010

Kronrod, Y., Coppess, E., \& Feldman, N. H. (2016). A unified account of categorical effects in phonetic perception. Psychonomic Bulletin \& Review, 23(6), 1681-1712. doi:10.3758/s13423-016-1049-y

Kuhl, P. K., Williams, K. A., Lacerda, F., Stevens, K. N., \& Lindblom, B. (1992). Linguistic experience alters phonetic perception in infants by 6 months of age. Science, 255, 606-8.

Lisker, L., \& Abramson, A. (1964). A cross-language sudy of voicing in initial stops: Acoustical measurements. Word, 20(3), 384-422.

Marr, D. (1982). Vision: A computational investigation into the human representation and processing of visual information. New York, NY, USA: Henry Holt and Co., Inc.

Maye, J., \& Gerken, L. (2000). Learning Phonemes Without Minimal Pairs. In S. C. Howell (Ed.), BUCLD 24 Proceedings (pp. 522-533). Somerville, MA: Cascadilla Press.

Maye, J., Werker, J. F., \& Gerken, L. (2002). Infant sensitivity to distributional information can affect phonetic discrimination. Cognition, 82(3), B101-11. PMID: 11747867 
McMurray, B., Aslin, R. N., \& Toscano, J. C. (2009). Statistical learning of phonetic categories: Insights from a computational approach. Developmental Science, 12(3), 369-378. doi:10.1111/j.1467-7687.2009. 00822.x

Meschiari, S. (2015). Latex2exp: Use latex expressions in plots. R package version 0.4.0. Retrieved from https://CRAN.R-project.org/package=latex2exp

Miyawaki, K., Jenkins, J. J., Strange, W., Liberman, A. M., Verbrugge, R., \& Fujimura, O. (1975). An effect of linguistic experience: The discrimination of [r] and [1] by native speakers of Japanese and English. Perception \& Psychophysics, 18(5), 331-340. doi:10.3758/BF03211209

Munson, C. M. (2011). Perceptual learning in speech reveals pathways of processing.

Newman, R. S., Clouse, S. A., \& Burnham, J. L. (2001). The perceptual consequences of within-talker variability in fricative production. The Journal of the Acoustical Society of America, 109(3), 11811196. doi: $10.1121 / 1.1348009$

Nielsen, K., \& Wilson, C. (2008). A Hierarchical Bayesian Model of Multi-level Phonetic Imitation. In Proceedings of the 27th West Coast Conference on Formal Linguistics (pp. 335-343).

Norris, D., McQueen, J. M., \& Cutler, A. (2003). Perceptual learning in speech. Cognitive Psychology, 47(2), 204-238. doi:10.1016/S0010-0285(03)00006-9

Olasagasti, I., \& Giraud, A.-L. (2018). Integrating prediction errors at two time scales permits rapid recalibration of speech sound categories. bioRxiv, 479089. doi:10.1101/479089

Qian, T., Jaeger, T. F., \& Aslin, R. N. (2012). Learning to represent a multi-context environment: More than detecting changes. Frontiers in Psychology, 3, 228-228. doi:10.3389/fpsyg.2012.00228

R Core Team. (2020). R: A language and environment for statistical computing. R Foundation for Statistical Computing. Vienna, Austria. Retrieved from https://www.R-project.org/

Saltzman, D., \& Myers, E. (2017). Listeners are maximally flexible in updating phonetic beliefs over time. Psychonomic Bulletin \& Review. doi:10.3758/s13423-017-1376-7

Sanborn, A. N., Griffiths, T. L., \& Navarro, D. J. (2010). Rational approximations to rational models: Alternative algorithms for category learning. Psychological Review, 117(4), 1144-67. doi:10.1037/ a0020511. PMID: 21038975

Schertz, J., \& Kang, Y. (2016). Variation's variable influence on perception: How talker information affects cross-dialectal Korean phonetic categorization. Manuscript submitted for publication.

Schreiber, E., Onishi, K., \& Clayards, M. A. (2013). Manipulating phonological boundaries using distributional cues. In Proceedings of Meetings on Acoustics (Vol. 19). doi:10.1121/1.4801082

Schuster, S., \& Degen, J. (2020). Speaker-specific adaptation to variable use of uncertainty expressions. In A. Goel, C. Seifert, \& C. Freksa (Eds.), Proceedings of the 41st Annual Conference of the Cognitive Science Society, Austin, TX: Cognitive Science Society.

Schweinhart, A. M., Shafto, P., \& Essock, E. A. (2017). Distribution of content in recently-viewed scenes whitens perception. Journal of Vision, 17(3), 8-8. doi:10.1167/17.3.8

Simoncelli, E. P., \& Olshausen, B. A. (2001). Natural image statistics and neural representation. Annual Review of Neuroscience, 24(1), 1193-216. doi:10.1146/annurev.neuro.24.1.1193. PMID: 11520932

Stocker, A. A., \& Simoncelli, E. P. (2006). Sensory Adaptation within a Bayesian Framework for Perception. In Y. Weiss, B. Schölkoph, \& J. Platt (Eds.), Advances in Neural Information Processing Systems (Vol. 18, pp. 1291-1298). MIT Press.

Sumner, M. (2011). The role of variation in the perception of accented speech. Cognition, 119(1), 131-6. doi:10.1016/j.cognition.2010.10.018. PMID: 21144500

Takagi, N., \& Mann, V. (1995). The limits of extended naturalistic exposure on the perceptual mastery of English /r/ and /l/ by adult Japanese learners of English. Applied Psycholinguistics, 16(4), 380-406. doi:10.1017/S0142716400066005

Tavoni, G., Balasubramanian, V., \& Gold, J. I. (2019). What is optimal in optimal inference? Current Opinion in Behavioral Sciences, 29, 117-126. doi:10.1016/j.cobeha.2019.07.008

Theodore, R. M., \& Monto, N. R. (2019). Distributional learning for speech reflects cumulative exposure to a talker's phonetic distributions. Psychonomic Bulletin \& Review. doi:10.3758/s13423-018-1551-5

Theodore, R. M., Myers, E. B., \& a. Lomibao, J. (2015). Talker-specific influences on phonetic category structure. The Journal of the Acoustical Society of America, 138(2), 1068-1078. doi:10.1121/1.4927489 
Vallabha, G. K., McClelland, J. L., Pons, F., Werker, J. F., \& Amano, S. (2007). Unsupervised learning of vowel categories from infant-directed speech. Proceedings of the National Academy of Sciences of the United States of America, 104(33), 13273-8. doi:10.1073/pnas.0705369104

Vehtari, A., Gabry, J., Magnusson, M., Yao, Y., \& Gelman, A. (2019). Loo: Efficient leave-one-out crossvalidation and waic for bayesian models. $\mathrm{R}$ package version 2.2.0. Retrieved from https://CRAN.Rproject.org $/$ package $=$ loo

Vehtari, A., Gelman, A., \& Gabry, J. (2017). Practical Bayesian model evaluation using leave-one-out crossvalidation and WAIC. Statistics and Computing, 27(5), 1413-1432. doi:10.1007/s11222-016-9696-4

Vroomen, J., van Linden, S., de Gelder, B., \& Bertelson, P. (2007). Visual recalibration and selective adaptation in auditory-visual speech perception: Contrasting build-up courses. Neuropsychologia, 45(3), 572-7. doi:10.1016/j.neuropsychologia.2006.01.031. PMID: 16530233

Werker, J. F., \& Tees, R. C. (1984). Cross-language speech perception: Evidence for perceptual reorganization during the first year of life. Infant Behavior and Development, 7(1), 49-63. doi:10.1016/S0163-6383(84) 80022-3

Wichmann, F. A., \& Hill, N. J. (2001). The psychometric function: I. Fitting, sampling, and goodness of fit. Perception \& psychophysics, 63(8), 1293-313. PMID: 11800458

Wickham, H. (2017). Tidyverse: Easily install and load the 'tidyverse'. R package version 1.2.1. Retrieved from https://CRAN.R-project.org/package $=$ tidyverse

Wickham, H. (2018). Forcats: Tools for working with categorical variables (factors). R package version 0.3.0. Retrieved from https://CRAN.R-project.org/package=forcats

Wickham, H., Chang, W., Henry, L., Pedersen, T. L., Takahashi, K., Wilke, C., \& Woo, K. (2018). Ggplot2: Create elegant data visualisations using the grammar of graphics. $\mathrm{R}$ package version 3.0.0. Retrieved from https://CRAN.R-project.org/package=ggplot2

Wickham, H., François, R., Henry, L., \& Müller, K. (2018). Dplyr: A grammar of data manipulation. R package version 0.7.6. Retrieved from https://CRAN.R-project.org/package=dplyr

Wilke, C. O. (2018). Cowplot: Streamlined plot theme and plot annotations for 'ggplot2'. R package version 0.9.3. Retrieved from https://CRAN.R-project.org/package=cowplot

Xie, Y. (2018). Knitr: A general-purpose package for dynamic report generation in $r$. R package version 1.20. Retrieved from https://CRAN.R-project.org/package $=$ knitr

Yildirim, I., Degen, J., Tanenhaus, M. K., \& Jaeger, T. F. (2016). Talker-specificity and adaptation in quantifier interpretation. Journal of Memory and Language, 87, 128-143. doi:10.1016/j.jml.2015.08.003 